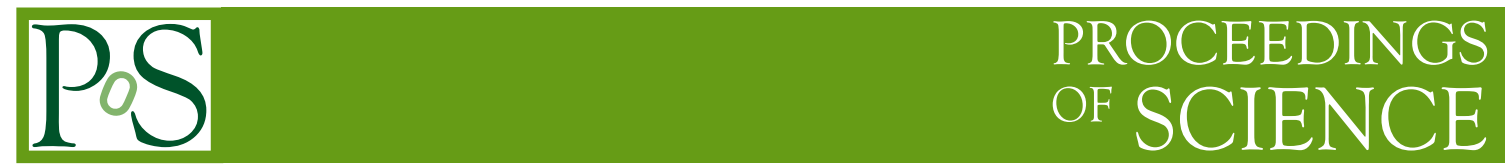

\title{
Introduction to Conformal Field Theory
}

\author{
Antonin Rovai ${ }^{* \dagger}$ \\ Université Libre de Bruxelles and International Solvay Institues \\ E-mail: antonin.rovaiculb.ac.be
}

An elementary introduction to Conformal Field Theory is provided. We start by reviewing symmetries in classical and quantum field theories and the associated existence of conserved currents by Noether's theorem. Next we describe general facts on conformal invariance in $d$ dimensions and finish with the analysis of the special case $d=2$, including an introduction to the operator formalism and a discussion of the trace anomaly.

Eighth Modave Summer School in Mathematical Physics

26th august - Ist september 2012

Modave, Belgium

* Speaker.

${ }^{\dagger}$ FNRS Research Follow 


\section{Contents}

Foreword 2

$\begin{array}{ll}\text { Introduction } & 3\end{array}$

1. Symmetries and Conservation laws 3

1.1 Definitions 4

$\begin{array}{ll}1.2 & \text { Noether's theorem }\end{array}$

1.3 The energy-momentum tensor 8

$\begin{array}{ll}1.4 \text { Consequences for the quantum theory } & 10\end{array}$

2. Conformal invariance in $d$ dimensions 11

2.1 General considerations and algebra 11

$\begin{array}{ll}2.2 & \text { Action of conformal transformations on fields } \\ \end{array}$

$\begin{array}{lll}2.3 & \text { Energy-momentum tensor and conformal invariance } & 16\end{array}$

2.4 Conformal invariance in quantum field theory and Ward identities 16

3. Conformal invariance in two dimensions 19

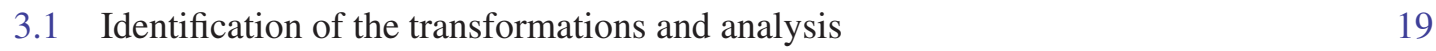

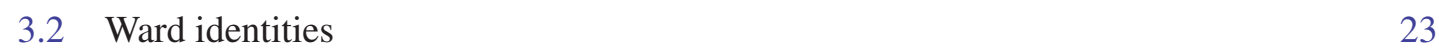

3.3 Operator Formalism 26

3.3.1 Radial order and mode expansion 26

3.3.2 Operator Product Expansion 27

$\begin{array}{lll}3.3 .3 & \text { Virasoro algebra } & 29\end{array}$

3.4 Trace anomaly 30

References

\section{Foreword}

These lectures notes were written for the eighth edition of the Modave Summer School in Mathematical Physics. During this edition, there were several lectures [1,2] relying on Conformal Field Theory basics, and thus self-consistency of the school required an elementary introduction to this vast subject. The present lectures were scheduled at the very beginning of the school, right after the gong show on the first day and in the morning of the second day. People were then smoothly taken from very basics to advanced research topics throughout the week, overall in the relaxed atmosphere of the charming village of Modave, Belgium. This eighth edition was a real success, and I hope this school will continue to exist for many more years to come. 


\section{Introduction}

These lecture notes consist of an elementary introduction to Conformal Field Theory (CFT). This class of theories have the property that they are invariant under so called conformal transformations, that may be seen as local scale tranformations. Conformal invariance is a vast topic, and in these notes we will be able to cover only the very basic elements. Historically, conformal invariance was first used to study statistical systems at a critical point, where scale invariance follows from the divergence of the correlation length. Since then, the use of CFTs has spread to the field of fundamental interactions: it is at the heart of string theory, the world-sheet theory being itself a two-dimensional CFT; in two dimensions, when quantum gravity is coupled to a matter CFT, the resulting effective gravitational action is the Liouville action which is a CFT; finally, the best understood example of a gauge/gravity duality that we know of involves a CFT on the field theory side, namely $\mathscr{N}=4$ super-Yang-Mills in four dimensions.

The plan of these notes is as follows. We start very slowly in section 1 with basic results concerning symmetries in field theory, both at the classical and at the quantum level. The discussion will be quite general and several examples will be presented, with special emphasis on the energymomentum tensor, which play a crucial rôle in the following. In section 2, we introduce and study conformal transformations in $d$ dimensions. We discuss finite transformations as well as the algebra of the conformal group, and we explore some simple consequences of conformal invariance on twoand three-point functions. Finally in section 3 we focus on the special case of $d=2$. We introduce primary fields, study Ward identities and the associated consequences for the energy-momentum tensor, operator formalism and operator product expansion. We end these lectures with a discussion of the trace anomaly.

For (much) more details on Conformal Field Theory, we refer the interested reader to the abundant literature. The standard reference for a modern treatment is the very nice book by Di Francesco, Mathieu and Sénéchal [3], on which most of the present lectures is based. See also the lecture notes by Ginsparg [4]. For a more old-fashioned presentation, see the lectures by Coleman [5]. Note also that Polchinski's book [6] and Tong's lecture notes [7] on String Theory both include a quick introduction to conformal invariance in two dimensions. See also the chapter 15 in [8] for a nice introduction to more advanced topics.

Our conventions are the following: unless explicitly stated, we work in the euclidean $d$ dimensional flat space $\mathbf{R}^{d}$. Space-time points are denoted by $\mathbf{x}=\left(x^{\mu}\right)$ with $\mu=1, \ldots, d$, the last component $x^{d}$ being Euclidean time (except at the end of chapter 3, from section 3.3.1). Vector and form indices are lowered and raised using the flat metric, and we sum over repeated indices. We use natural units in which $c=\hbar=1$ and we use the symbol $\equiv$ for an equality valid by definition.

\section{Symmetries and Conservation laws}

Symmetries lie at the heart of our modern conception of physics. It is therefore very important to understand how we formulate the symmetry properties of a given theory and their consequences on observables. In this section we quickly review some elementary notions along these lines, preparing the ground for the analysis of conformal invariance. 


\subsection{Definitions}

We consider a classical theory for some fields, collectively denoted as $\Phi$, which are functions on a space-time manifold that we shall take to be flat $\mathbf{R}^{d}$. The dynamics of the fields $\Phi$ is fixed by a Lagrangian density $\mathscr{L}\left(\Phi, \partial_{\mu} \Phi\right)$ or by the action $S[\Phi]$ defined by

$$
S[\Phi]=\int \mathrm{d}^{d} x \mathscr{L}\left(\Phi, \partial_{\mu} \Phi\right) .
$$

Consider a map $\mathbf{x} \mapsto \mathbf{x}^{\prime}(\mathbf{x})$, where $\mathbf{x}^{\prime} \in \mathbf{R}^{d}$ is some invertible function of $\mathbf{x} \in \mathbf{R}^{d}$, together with some transformation of the fields $\Phi \mapsto \Phi^{\prime}$ defined by

$$
\Phi^{\prime}\left(\mathbf{x}^{\prime}\right)=F(\Phi(\mathbf{x}))
$$

for some function $F$. Under such a transformation, the action will generally be modified: $S \mapsto S^{\prime}$, with $S^{\prime}$ defined by the equation $S^{\prime}\left[\Phi^{\prime}\right]=S[\Phi]$, and the transformation is a symmetry if $S=S^{\prime}$.

Let us consider some examples.

1. Translations are simply defined by

$$
\mathbf{x}^{\prime}=\mathbf{x}+\mathbf{a},
$$

where $\mathbf{a} \in \mathbf{R}^{d}$. Most of the fields $\Phi$ that we consider are scalars under translation, that is, $F$ reduces to the identity:

$$
F(\Phi(\mathbf{x}))=\Phi(\mathbf{x})=\Phi^{\prime}\left(\mathbf{x}^{\prime}\right)=\Phi^{\prime}(\mathbf{x}+\mathbf{a})
$$

2. Rotations are given by

$$
x^{\prime \mu}=R_{v}^{\mu} x^{v}
$$

where the matrix $R$ is such that

$$
\delta_{\mu v} R_{\lambda}^{\mu} R_{\rho}^{v}=\delta_{\lambda \rho}
$$

The function $F$ corresponding to rotations is characterised by the representation that we choose for the field $\Phi$. For example, for a scalar field $\varphi$, the transformation is

$$
\varphi^{\prime}(R \cdot \mathbf{x})=\varphi(\mathbf{x})
$$

where we use the common notation $(R \cdot x)^{\mu}=R^{\mu}{ }_{v} x^{v}$. For a vector field $V^{\mu}$ the transformation is

$$
V^{\prime \mu}(R \cdot \mathbf{x})=R^{\mu}{ }_{v} V^{v}(\mathbf{x}),
$$

and so on for tensors of various ranks. In the quantum theory, we are also interested in spinor representations of the rotation group, that is, representations up to a phase. We shall however continue to write "representation of rotations" instead of "representation of spinor representation of the rotation group."

For a field $\Phi$ transforming in any representation $L$ of the rotation group, we write the corresponding transformation function $F$ as

$$
\Phi^{\prime}(R \cdot \mathbf{x})=L_{R}[\Phi(\mathbf{x})]
$$


that is, $L_{R}$ is the linear operator representing the transformation $R$.

Note that in Minkowskian space-time, we are more interested in transformations that preserve the Minkowski metric $\eta_{\mu v}$, namely the Lorentz transformations. As far as these lectures are concerned, the differences between the rotation group and the Lorentz group will not be important.

3. Scale transformations (or dilatations) read

$$
\mathbf{x}^{\prime}=\lambda \mathbf{x}
$$

where $\lambda \neq 0$. We define $\Phi^{\prime}$ by

$$
\Phi^{\prime}(\lambda \mathbf{x})=\lambda^{-\Delta} \Phi(\mathbf{x})
$$

where the number $\Delta$ is called the scaling dimension of $\Phi$. In this case the jacobian of the transformation is $\left|\partial \mathbf{x}^{\prime} / \partial \mathbf{x}\right|=\lambda^{d}$ and hence

$$
\begin{aligned}
S^{\prime}\left[\Phi^{\prime}\right] & =\int \mathrm{d}^{d} x^{\prime} \mathscr{L}\left(\Phi^{\prime}\left(\mathbf{x}^{\prime}\right), \partial_{\mu}^{\prime} \Phi^{\prime}\left(\mathbf{x}^{\prime}\right)\right) \\
& =\lambda^{d} \int \mathrm{d}^{d} x \mathscr{L}\left(\lambda^{-\Delta} \Phi(\mathbf{x}), \lambda^{-1-\Delta} \partial_{\mu} \Phi(\mathbf{x})\right) .
\end{aligned}
$$

In the case of a free theory for a massless scalar field $\varphi, \mathscr{L}$ is given by

$$
\mathscr{L}\left(\varphi, \partial_{\mu} \varphi\right)=\frac{1}{2} \partial_{\mu} \varphi \partial^{\mu} \varphi
$$

and the action $S$ is invariant if and only if the scaling dimension $\Delta$ is given by

$$
\Delta=\frac{d-2}{2} .
$$

\section{Scaling dimension versus mass dimension}

As far as classical field theory is concerned, the value of $\Delta$ for a given field will always coincide with the opposite of its mass dimension, itself determined by the requirement that the action is dimensionless (in units where $c=\hbar=1$ that we shall use throughout these notes). It should however be clear that the requirement of scale invariance is not a consequence of the fact that the action is dimensionless, since under a dilatation, only fields and coordinates are changed, whereas all other quantities (like coupling constants or masses) are held fixed. For instance, if we add a mass term $m^{2} \varphi^{2} / 2$ to the lagrangian (1.14) for the scalar field $\varphi$, then under the dilatation $\mathbf{x}^{\prime}=\lambda \mathbf{x}$ the transformed action (1.12) is given by

$$
S^{\prime}\left[\varphi^{\prime}\right]=\int \mathrm{d}^{d} x\left(\frac{1}{2} \partial_{\mu} \varphi \partial^{\mu} \varphi+\lambda^{2} \frac{m^{2}}{2} \varphi^{2}\right) \neq S[\varphi] .
$$

Mass terms are thus prohibited in dilatation invariant theories, as well as dimensionful coupling constants. 


\subsection{Noether's theorem}

Let us consider a continuous transformation, that is, the map $\mathbf{x} \mapsto \mathbf{x}^{\prime}$ is characterised continuously by some parameters $\omega_{a}$. We can then consider a transformation "close to identity," that is, for

$$
\mathbf{x}^{\prime}=\mathbf{x}+\omega_{a} \frac{\delta \mathbf{x}}{\delta \omega_{a}}
$$

we can write

$$
F(\Phi(\mathbf{x}))=\Phi(\mathbf{x})+\omega_{a} \frac{\delta F(\Phi(\mathbf{x}))}{\delta \omega_{a}}
$$

where summation on the index $a$ is understood. We define the generators $G_{a}$ by

$$
\delta_{\omega} \Phi(\mathbf{x})=\Phi^{\prime}(\mathbf{x})-\Phi(\mathbf{x})=-i \omega_{a} G_{a} \Phi(\mathbf{x}),
$$

and hence

$$
i G_{a} \Phi=\frac{\delta x^{\mu}}{\delta \omega_{a}} \partial_{\mu} \Phi-\frac{\delta F}{\delta \omega_{a}} .
$$

The factor of $i$ in the definition (1.19) is introduced for later convenience.

We now identify the parameters and the generators in our examples.

1. Translations: the parameters $\omega_{a}$ for an infinitesimal translation are the components $a_{\mu}$ of the infinitesimal vector a defining the infinitesimal transformation, and thus the index $a$ is in this case a space-time index $\mu: \omega_{\mu}=a_{\mu}$. Using (1.4) we thus find that

$$
\frac{\delta x^{\mu}}{\delta \omega_{v}}=\delta^{\mu v}, \quad \frac{\delta F}{\delta \omega_{\mu}}=0 .
$$

The generator, that we write $P_{\mu}$ and define by equation (1.20), reads

$$
P_{\mu}=-i \partial_{\mu}
$$

2. A infinitesimal rotation is characterised by an antisymmetric matrix $\omega_{\mu v}=-\omega_{v \mu}$ and is given by

$$
x^{\prime \mu}=x^{\mu}+\omega^{\mu}{ }_{v} x^{\nu} .
$$

Formula (1.17) then yields the following variation:

$$
\frac{\delta x^{\mu}}{\delta \omega_{v \rho}}=\frac{1}{2}\left(\delta^{\mu v} x^{\rho}-\delta^{\mu \rho} x^{v}\right) .
$$

For a field $\Phi$ transforming under a general representation $L$ as in (1.9), the effect of an infinitesimal rotation is of the form

$$
L_{R}[\Phi]=\Phi-\frac{i}{2} \omega_{\mu v} S^{\mu v}[\Phi]
$$

for some operators $S^{\mu v}=-S^{v \mu}$ representing the rotation algebra, the numerical factors being introduced for future convenience. Using (1.24) and (1.25), the generators $L^{\mu v}$ for rotations and defined in (1.20) are thus given by

$$
L^{\mu v}=i\left(x^{\mu} \partial^{v}-x^{v} \partial^{\mu}\right)+S^{\mu v} .
$$


3. For scale transformations $\mathbf{x} \mapsto \mathbf{x}^{\prime}=\lambda \mathbf{x}$, the transformation parameter is $\lambda$. We write $\lambda=1+\varepsilon$ for small $\varepsilon$, and equations (1.17) and (1.18) then gives $\delta x^{\prime \mu} / \delta \varepsilon=x^{\mu}$. Equation (1.18) then yields

$$
F(\Phi(\mathbf{x}))=\Phi^{\prime}\left(\mathbf{x}^{\prime}\right)=(1-\Delta \varepsilon) \Phi(\mathbf{x}),
$$

where we used $\delta F / \delta \varepsilon=-\Delta$ which is a consequence of the definition (1.11). If we call $D$ the dilatation generator, then (1.27) yields

$$
i D=\mathbf{x} \cdot \partial+\Delta,
$$

where we used the convenient notation $\mathbf{x} \cdot \partial=x^{\mu} \partial_{\mu}$.

For any continuous transformation, we can define the associated canonical current densities $j_{a}^{\mu}$ by

$$
j_{a}^{\mu}=\left(\frac{\partial \mathscr{L}}{\partial\left(\partial_{\mu} \Phi\right)} \partial_{v} \Phi-\delta_{v}^{\mu} \mathscr{L}\right) \frac{\delta x^{v}}{\delta \omega_{a}}-\frac{\partial \mathscr{L}}{\partial\left(\partial_{\mu} \Phi\right)} \frac{\delta F}{\delta \omega_{a}} .
$$

When the transformation under consideration is a symmetry and when the equations of motion are satisfied, the currents (1.29) are conserved,

$$
\partial_{\mu} j_{a}^{\mu}=0
$$

To show this, it is useful to assume for a moment that the parameters $\omega_{a}$ are arbitrary (but small) space-time functions $\omega_{a}(\mathbf{x})$. Using the formula $\operatorname{det}(1+J)=1+\operatorname{tr} J+O(J)^{2}$ where $J=\partial \mathbf{x}^{\prime} / \partial \mathbf{x}$ is the jacobian matrix for the map $\mathbf{x} \mapsto \mathbf{x}^{\prime}$, we find

$$
\left|\frac{\partial \mathbf{x}^{\prime}}{\partial \mathbf{x}}\right|=1+\partial_{\mu}\left(\omega_{a} \frac{\delta x^{\mu}}{\delta \omega_{a}}\right)+O(\omega)^{2}
$$

Working to first order in $\omega_{a}$, we find after some algebra the following formula for the variation $\delta S(\Phi) \equiv S^{\prime}(\Phi)-S(\Phi)$

$$
\delta S(\Phi)=\int \mathrm{d}^{d} \mathbf{x}\left(\partial_{\mu} \omega_{a} \frac{\delta x^{\mu}}{\delta \omega_{a}} \mathscr{L}-\frac{\partial \mathscr{L}}{\partial\left(\partial_{\mu} \phi\right)} \partial_{\mu} \omega_{a} \frac{\delta x^{\rho}}{\delta \omega_{a}} \partial_{\rho} \Phi+\frac{\partial \mathscr{L}}{\partial\left(\partial_{\mu} \Phi\right)} \partial_{\mu} \omega_{a} \frac{\delta F}{\delta \omega_{a}}\right) .
$$

Dropping some total derivative terms, we find that

$$
\delta S=\int \mathrm{d}^{d} x \partial_{\mu} j_{a}^{\mu}(\mathbf{x}) \omega(\mathbf{x}),
$$

where the currents $j_{a}^{\mu}$ are given by (1.29). When the equations of motion are satisfied, the action is by definition stationary under arbitrary variations and thus $\delta S=0$. Since the functions $\omega_{a}$ in (1.33) are arbitrary, this is possible only if $\partial_{\mu} j_{a}^{\mu}=0$, completing the proof.

To any current $j_{a}^{\mu}$ we associate a conserved charge $Q_{a}$ by the formula

$$
Q_{a}=\int_{\Sigma} \mathrm{d}^{d-1} x j_{a}^{d},
$$

where $\Sigma$ is the $x^{d}=$ constant hyper surface. When the current $j_{a}^{\mu}$ is conserved, the charge $Q_{a}$ is also conserved in the sense that $\mathrm{d} Q_{a} / \mathrm{d} x^{d}=0$. Note that several currents may give rise to the same charge. For example, if we define

$$
\tilde{j}_{a}^{\mu}=j_{a}^{\mu}+\partial_{v} B_{a}^{v \mu}
$$


for $B_{a}^{\mu v}=-B_{a}^{v \mu}$, then conservation of $j_{a}^{\mu}$ implies that $\tilde{j}_{a}^{\mu}$ is conserved as well. Moreover, the charge associated with $\tilde{j}_{a}^{\mu}$ coincides with $Q_{a}$, as the integral of $\partial_{v} B_{a}^{v d}=\partial_{i} B_{a}^{i d}$ over $\Sigma$ vanishes. Terms such as $\partial_{v} B_{a}^{v \mu}$ are called improvement terms. We will make use of such terms to define a symmetric stress-energy tensor, see equation (1.40).

\subsection{The energy-momentum tensor}

For many reasons, the energy-momentum (or stress) tensor plays a crucial rôle in quantum field theory, and in our case this will be shortly obvious. It is defined as the current corresponding to translation invariance of the theory. For fields $\Phi$ transforming as scalars under translations, $F$ reduce to the identity and thus

$$
T_{c}^{\mu}{ }_{v}=\frac{\partial \mathscr{L}}{\partial\left(\partial_{\mu} \Phi\right)} \partial_{v} \Phi-\delta_{v}^{\mu} \mathscr{L}
$$

where the subscript $c$ indicates that this is the canonical energy-momentum tensor. Notice that the index $a$ in the general definition of the current $j_{a}^{\mu}$ in (1.29) is now replaced by a space-time index $\mu$, since translations are parameterised by a space-time vector. The associated current $T_{c}{ }^{\mu}{ }_{v}$, defined in (1.36), thus carries two space-time indices.

The associated conserved charges also carry a space-time index, and are the components of the energy-momentum vector $\mathrm{P}^{\mu}$ :

$$
\mathrm{P}^{\mu}=\int_{\Sigma} \mathrm{d}^{d-1} x T_{c}^{d \mu}
$$

For future reference, let us quickly write the currents associated to Lorentz and dilatation invariance. From the definition (1.29) and the formulas derived in the examples of sections 1.1 and 1.2 , we find the following expressions for the currents:

$$
\begin{array}{rlr}
j^{\mu v \rho} & =T_{c}^{\mu v} x^{\rho}-T_{c}^{\mu \rho} x^{v}+i \frac{\partial \mathscr{L}}{\partial\left(\partial_{\mu} \Phi\right)} S^{v \rho} \Phi & \text { (rotations) }, \\
j_{D}^{\mu} & =T_{c}{ }^{\mu}{ }_{v} x^{v}+\frac{\partial \mathscr{L}}{\partial\left(\partial_{\mu} \Phi\right)} \Delta \Phi & \text { (dilatations). }
\end{array}
$$

In general, the canonical energy-momentum tensor $T_{c}^{\mu v}$ is not symmetric under the exchange of $\mu$ with $v$. In a Lorentz invariant theory however, it is however always possible to define a symmetric energy-momentum tensor by adding to $T_{c}^{\mu v}$ a suitable improvement term, following the discussion below (1.35). The resulting symmetric energy-momentum tensor $T_{B}^{\mu v}$ is called the Belinfante stress tensor, and is given by

$$
T_{B}^{\mu v}=T_{c}^{\mu v}+\partial_{\rho} B^{\rho \mu v}
$$

where the improvement term $B^{\rho \mu v}$ defined by

$$
B^{\mu \rho v}=\frac{i}{2}\left[\frac{\partial \mathscr{L}}{\partial\left(\partial_{\mu} \Phi\right)} S^{v \rho} \Phi+\frac{\partial \mathscr{L}}{\partial\left(\partial_{\rho} \Phi\right)} S^{\mu v} \Phi+\frac{\partial \mathscr{L}}{\partial\left(\partial_{v} \Phi\right)} S^{\mu \rho} \Phi\right]
$$

The new currents $T_{B}^{\mu v}$ are conserved since $B^{\rho \mu \nu}$ is antisymmetric under the exchange of $\rho$ and $\mu$ thanks to the fact that the Lorentz generators $S^{\mu \nu}$ are themselves antisymmetric. Moreover, the current (1.38) associated to Lorentz invariance can be written as

$$
j^{\mu v \rho}=T_{B}^{\mu v} x^{\rho}-T_{B}^{\mu \rho} x^{v} .
$$


As a consequence, the constraints $\partial_{\mu} j^{\mu v \rho}=0$ and $\partial_{\mu} T_{B}^{\mu v}=0$ imply the symmetry of $T_{B}^{\mu v}$ :

$$
T_{B}^{\mu v}=T_{B}^{v \mu}
$$

Let us remark that the Belinfante stress tensor $T_{B}{ }^{\mu v}$ is symmetric only when the equations of motion are satisfied, because we used the conservation laws $\partial_{\mu} j^{\mu v \rho}=0$ and $\partial_{\mu} T_{B}^{\mu v}=0$ to show (1.43).

\section{An alternative definition of the energy-momentum tensor}

We now motivate an alternative definition of the momentum tensor that is automatically symmetric and generalises to curved space-times. Assume the energy-momentum tensor $T^{\mu v}$ has been made symmetric. Under an arbitrary infinitesimal change of coordinates

$$
\mathbf{x} \mapsto \mathbf{x}+\varepsilon(\mathbf{x}),
$$

the infinitesimal variation of the action $\delta S$ is given by (1.32),

$$
\delta S=-\int \mathrm{d}^{d} x T^{\mu v} \partial_{\mu} \varepsilon_{v}=-\frac{1}{2} \int \mathrm{d}^{d} x T^{\mu v}\left(\partial_{\mu} \varepsilon_{v}+\partial_{\nu} \varepsilon_{\mu}\right) .
$$

Now consider the generalization of this theory on a curved space-time of metric $g$, and let $S[\Phi ; g]$ be a diffeomorphism invariant action such that it reduces to our original action $S$ when restricted on flat space ${ }^{1}$ where the metric $g$ is the identity matrix that we write $\delta$,

$$
S[\Phi ; \delta]=S[\Phi]
$$

The action $S[\Phi ; g]$ is by definition invariant under the infinitesimal diffeomorphism (1.44). Evaluating its variation $\delta S$ on flat space, the requirement $\delta S[\Phi, g]=0$ implies

$$
\left.\delta S[\Phi ; g]\right|_{g=\delta}=\int \mathrm{d}^{d} x\left(\left.\frac{\delta S[\Phi ; g]}{\delta g_{\mu v}}\right|_{g=\delta} \delta g_{\mu v}-\frac{1}{2} T^{\mu v}\left(\partial_{\mu} \varepsilon_{v}+\partial_{\nu} \varepsilon_{\mu}\right)\right)=0 .
$$

Plugging the variation of the metric around flat space $\delta g_{\mu \nu}=-\partial_{\mu} \varepsilon_{v}-\partial_{\nu} \varepsilon_{\mu}$ into (1.47) yields

$$
T^{\mu v}=-\left.2 \frac{\delta S[\Phi ; g]}{\delta g_{\mu \nu}}\right|_{g=\delta} .
$$

Formula (1.48) suggest the following definition for the energy-momentum tensor in curved spacetimes:

$$
T^{\mu v}(g) \equiv-\frac{2}{\sqrt{g}} \frac{\delta S[\Phi ; g]}{\delta g_{\mu v}} \quad \text { (curved space-time), }
$$

the factor involving the determinant of the metric being introduced in order to have a tensor (rather than a density). Loosely speaking, the energy-momentum tensor captures the variation of the theory under an arbitrary variation of the metric. Notice that it is formula (1.49) that is used to define the energy-momentum tensor in general relativity, where $T_{\mu \nu}$ appears as a source term in Einstein's equations.

\footnotetext{
${ }^{1}$ The action $S[\Phi ; g]$ is not uniquely defined by the condition (1.46), as any term that vanishes on flat space may be added to it.
} 


\subsection{Consequences for the quantum theory}

We now study symmetries in quantum field theories. Since observables are typically expressed in terms of correlation functions of the fields (or operators) of the theory, it is natural to define a symmetry of a quantum theory under some transformation $\mathbf{x} \mapsto \mathbf{x}^{\prime}$ with the associated transformation of fields as a condition on correlators. To be specific, the quantum theory is said to be invariant under $\mathbf{x} \mapsto \mathbf{x}^{\prime}, \Phi \mapsto \Phi^{\prime}$ with $\Phi^{\prime}\left(\mathbf{x}^{\prime}\right)=F(\Phi(x))$ if and only if

$$
\left\langle\Phi_{1}\left(\mathbf{x}_{1}^{\prime}\right) \cdots \Phi_{n}\left(\mathbf{x}_{n}^{\prime}\right)\right\rangle=\left\langle F\left(\Phi_{1}\left(\mathbf{x}_{1}\right)\right) \cdots F\left(\Phi_{n}\left(\mathbf{x}_{n}\right)\right)\right\rangle,
$$

where $\Phi_{1}\left(\mathbf{x}_{1}\right), \ldots, \Phi_{n}\left(\mathbf{x}_{n}\right)$ are fields (or operators) of the theory evaluated at some space-time points $\mathbf{x}_{1}, \ldots, \mathbf{x}_{n} \in \mathbf{R}^{d}$.

It should be kept in mind that if the classical theory has some symmetry, then in general this does not mean that the quantized theory also has this symmetry, in the sense of (1.50). When the symmetry is broken quantum mechanically, we say that the classical symmetry is anomalous. We shall encounter an example of anomaly in section 3.4. In this section (and in most of these lectures), we assume that the symmetries that we consider are not anomalous.

Let us write the infinitesimal version of the condition (1.50) for a continuous symmetry. Using the path-integral formalism for example, it is a simple matter to show that if $\omega_{a}$ is lifted to an arbitrary function $\omega_{a}(\mathbf{x})$, then

$$
\begin{aligned}
\delta\left\langle\Phi\left(\mathbf{x}_{1}\right) \cdots \Phi\left(\mathbf{x}_{n}\right)\right\rangle & =\int \mathrm{d}^{d} x \partial_{\mu}\left\langle j_{a}^{\mu}(\mathbf{x}) \Phi\left(\mathbf{x}_{1}\right) \cdots \Phi\left(\mathbf{x}_{n}\right)\right\rangle \omega_{a}(\mathbf{x}) \\
& =-i \int_{D} \mathrm{~d}^{d} x \omega_{a}(\mathbf{x}) \sum_{i=1}^{n}\left\langle\Phi\left(\mathbf{x}_{1}\right) \cdots G_{a} \Phi\left(\mathbf{x}_{i}\right) \cdots \Phi\left(\mathbf{x}_{n}\right)\right\rangle \delta^{d}\left(\mathbf{x}-\mathbf{x}_{i}\right),
\end{aligned}
$$

where the domain $D \subset \mathbf{R}^{d}$ contain all the points $\mathbf{x}_{i}, i=1, \ldots, n$ at which the fields are evaluated. Since this is valid for any value of the parameters $\omega_{a}$, we find the so-called Ward identities

$$
\frac{\partial}{\partial x^{\mu}}\left\langle j_{a}^{\mu}(\mathbf{x}) \Phi\left(\mathbf{x}_{1}\right) \cdots \Phi\left(\mathbf{x}_{n}\right)\right\rangle=-i \sum_{i=1}^{n} \delta^{d}\left(\mathbf{x}-\mathbf{x}_{i}\right)\left\langle\Phi\left(\mathbf{x}_{1}\right) \cdots G_{a} \Phi\left(\mathbf{x}_{i}\right) \cdots \Phi\left(\mathbf{x}_{n}\right)\right\rangle .
$$

Equations (1.52) are the quantum versions of the classical conservation law (1.30). Loosely speaking, one can say that in the quantum theory, the currents $j_{a}^{\mu}$ fail to be conserved when inserted in correlation functions because of the terms on the right hand side of (1.52). Such terms, that are non-zero (and singular) only when the current $j_{a}^{\mu}$ is evaluated at one of the points $\mathbf{x}_{i}$, are called contact terms.

Similarly to the classical case, the addition of an improvement term $\partial_{v} B_{a}^{\mu \nu}$ with $B_{a}^{\mu \nu}=-B_{a}^{v \mu}$ to the current $j_{a}^{\mu}$ as in (1.35), has no consequence on the Ward identities (1.52). Indeed, thanks to the antisymmetry property of $B_{a}^{\mu v}$ under the exchange on $\mu$ and $v$, we can replace $j_{a}^{\mu}$ by $\tilde{j}_{a}^{\mu}=$ $j_{a}^{\mu}+\partial_{v} B_{a}^{\mu \nu}$ into equation (1.52) without any further changes.

\section{Definition of the energy-momentum tensor in the quantum theory}

To define the energy-momentum tensor in the quantum theory on a general curved space-time, one could simply use the definition (1.49) as a statement between operators. However, if one wish to keep the spirit of this definition, equation (1.49) should be adapted because the classical action 
$S$ doesn't contain all the quantum effects of the theory. To state this more precisely, let us consider the path-integral expression for the partition function $Z[g]$,

$$
Z[g]=\int D \Phi_{g} e^{-S[\Phi ; g]} .
$$

As indicated explicitly in (1.53), the formal path-integral measure $D \Phi_{g}$ is metric-dependent, as a consequence of diffeomorphism invariance of the partition function $Z[g]$. For this reason, $Z[g]$ will receive essentially two contributions when the metric $g$ is perturbed: one from the usual variation of the action $S[g]$, and a new one from the variation of the measure $D \Phi_{g}$. Therefore, if one wish to keep the idea that the energy-momentum tensor captures the response of the theory under a perturbation of the metric, it is very natural to define it through the equation

$$
Z[g+\delta g]=\int D \Phi_{g+\delta g} e^{-S[\Phi ; g+\delta g]}=\int D \Phi_{g}\left(1+\frac{1}{2} \int \mathrm{d}^{d} x \sqrt{g} T^{\mu v} \delta g_{\mu v}\right) e^{-S[\Phi ; g]},
$$

where $\delta g$ is an arbitrary, small perturbation of the metric. If we define as usual the generating functional $W$ by

$$
W[g]=-\ln Z[g],
$$

then the definition of $T^{\mu v}$ is equivalent to

$$
\left\langle T^{\mu v}\right\rangle=-\frac{2}{\sqrt{g}} \frac{\delta W[g]}{\delta g_{\mu v}} .
$$

We will use equation (1.56) when we consider the trace anomaly in section 3.4, and it will also be very useful in other lectures of this school [1]. Formula (1.56) and (1.49) are quite analogous, but it should now be clear that in (1.56), the subtle quantum effects contained in the path-integral (1.53) have been taken into account. Of course, if we consider the classical limit of our theory, then the generating functional $W$ goes to the classical action $S$ of the theory, and we recover (1.49) from (1.56).

\section{Conformal invariance in $d$ dimensions}

In this section we define conformal transformations in any dimension $d$, and explore their basic properties.

\subsection{General considerations and algebra}

Consider a field theory on a $d$-dimensional space-time with metric $g$, that may be curved or not. A conformal transformation is a smooth, invertible map $\mathbf{x} \mapsto \mathbf{x}^{\prime}(\mathbf{x})$ such that

$$
\left.\left.\frac{\partial x^{\prime \rho}}{\partial x^{\mu}}\right|_{\mathbf{x}} \frac{\partial x^{\prime \lambda}}{\partial x^{v}}\right|_{\mathbf{x}} g_{\rho \lambda}\left(\mathbf{x}^{\prime}\right)=\Lambda(\mathbf{x}) g_{\mu \nu}(\mathbf{x}),
$$

together with some field transformation $\Phi \mapsto \Phi^{\prime}$ to be specified later and where $\Lambda$ is some strictly positive function.

Let us insist on the fact that under a conformal transformation, the metric is not changed, $g \mapsto g$. As a side remark, let us mention that conformal transformations are sometimes defined as 
diffeomorphisms that leave the metric unchanged up to a factor. This formulation is misleading since the metric is not changed under a conformal transformation, while for diffeomorphisms it is. Since we measure distances before and after a conformal transformation with the same metric, distances between points change, because the points have moved. On the other hand, distances are invariant under a diffeomorphism. Moreover, a diffeomorphism-invariant theory is clearly not automatically conformally invariant: conformal transformations are not a subset of diffeomorphisms.

We will now study the consequences of (2.1) on the map $\mathbf{x} \mapsto \mathbf{x}^{\prime}$ for flat space, $g_{\mu \nu}=\delta_{\mu \nu}$. The set of conformal transformations obviously form a group called the conformal group. Let us already note that this group should have the Euclidean group as a subgroup, ${ }^{2}$ as it corresponds to the special case $\Lambda(\mathbf{x})=1$. Otherwise, the Jacobian $\left|\partial \mathbf{x}^{\prime} / \partial \mathbf{x}\right|$ of the conformal transformation is $\Lambda(\mathbf{x})^{-d / 2}$, as can be seen by evaluating the determinant of both sides of equation (2.1).

Consider an infinitesimal transformation $\mathbf{x} \mapsto \mathbf{x}+\varepsilon(\mathbf{x})$. Setting $f=1-\Lambda$, we find the constraint

$$
\partial_{\mu} \varepsilon_{v}+\partial_{v} \varepsilon_{\mu}=f \delta_{\mu v}
$$

whose contraction with $\delta^{\mu v}$ yields

$$
f(\mathbf{x})=\frac{2}{d} \partial \cdot \varepsilon .
$$

If we apply $\partial_{\rho}$ on equation (2.2) we find

$$
\partial_{\rho \mu}^{2} \varepsilon_{v}+\partial_{\rho \nu}^{2} \varepsilon_{\mu}=\partial_{\rho} f \delta_{\mu \nu},
$$

which implies

$$
2 \partial_{\mu \nu}^{2} \varepsilon_{\rho}=\delta_{\mu \rho} \partial_{v} f+\delta_{v \rho} \partial_{\mu} f-\delta_{\mu \nu} \partial_{\rho} f .
$$

Contracting with $\delta^{\mu v}$, this gives

$$
2 \partial^{2} \varepsilon_{\mu}=(2-d) \partial_{\mu} f .
$$

Applying $\partial^{\mu}$ to this equation and combining with (2.3) yields

$$
(d-1) \partial^{2} f=0 .
$$

The case $d=1$ is trivial: any coordinate transformation obviously satisfies (2.1). The case $d=2$ is particular and will be studied in detail in the next section (although, as we will see then, some results derived below will also apply to $d=2$ ), and we focus on the case $d \geq 3$ from now on.

The equation $\partial_{\mu v}^{2} f=0$ is solved by

$$
f(\mathbf{x})=A+B_{\mu} x^{\mu},
$$

for some constants $A$ and $B_{\mu}$. This implies, using (2.6), that $\partial_{\mu \nu}^{2} \varepsilon$ is also a constant, and hence

$$
\varepsilon_{\mu}(\mathbf{x})=a_{\mu}+b_{\mu v} x^{v}+c_{\mu v \rho} x^{v} x^{\rho}
$$

for some constants $a_{\mu}, b_{\mu v}, c_{\mu v \rho}$ with $c_{\mu v \rho}=c_{\mu \rho v}$. Let us analyse these transformations case by case.

\footnotetext{
${ }^{2}$ In the Minkowskian case, the conformal group contain the Poincare group.
} 
1. $b_{\mu v}=0$ and $c_{\mu v \rho}=0$. Then $\varepsilon_{\mu}$ is a constant: these are just translations.

2. $a_{\mu}=0$ and $c_{\mu v \rho}=0$. In this case we find $f=2 b^{\mu}{ }_{\mu} / d$ and

$$
b_{\mu v}+b_{v \mu}=f \delta_{\mu v} .
$$

It is always possible to decompose $b_{\mu v}$ as its symmetric part $b_{(\mu v)}$ plus its antisymmetric part $b_{[\mu v]} \equiv m_{\mu v}$. Hence

$$
b_{\mu v}=\frac{b_{\rho}^{\rho}}{d} \delta_{\mu v}+m_{\mu v} .
$$

It is not difficult to see that the first term consists of an infinitesimal dilatation $\mathbf{x} \mapsto(1+\alpha) \mathbf{x}$, where $\alpha=b^{\mu}{ }_{\mu} / d$, while the last term corresponds to a rotation.

3. $a_{\mu}=0$ and $b_{\mu \nu}=0$. If we set $c_{\mu}=c^{\lambda}{ }_{\lambda \mu}$, then the various constraints imply

$$
c_{\rho \mu \nu}=\delta_{\mu \rho} c_{v}+\delta_{v \rho} c_{\mu}-\delta_{\mu \nu} c_{\rho},
$$

and so $\varepsilon^{\mu}(\mathbf{x})=2 x^{\mu} c_{v} x^{\nu}-c^{\mu} x^{2}$. This is a new transformation that is special to conformal transformations, and is therefore called a (infinitesimal) special conformal transformation (SCT for short).

We have now explored all possibilities for conformal transformations at the infinitesimal level. To find the finite transformations, we must exponentiate the different infinitesimal transformation that we just found. Although this is straightforward in principle, it can be tedious (in particular for the $\mathrm{SCT})$. The result is

$$
\begin{aligned}
x^{\prime \mu} & =x^{\mu}+a^{\mu} & & \text { (Translations) } \\
x^{\prime \mu} & =\lambda x^{\mu} & & \text { (Dilatations) } \\
x^{\prime \mu} & =R^{\mu}{ }_{v} x^{v} & & \text { (Rotations) } \\
x^{\prime \mu} & =\frac{x^{\mu}-b^{\mu} x^{2}}{1-2 b \cdot x+b^{2} x^{2}}, & & (\mathrm{SCT})
\end{aligned}
$$

where $\lambda, a^{\mu}$ and $b^{\mu}$ are numbers and $R^{\mu}{ }_{v}$ is a matrix that satisfies the constraint (1.6).

To get some intuition about an SCT, remark that under such a transformation $\mathbf{x} \mapsto \mathbf{x}^{\prime}$ we have

$$
\frac{x^{\prime \mu}}{x^{\prime 2}}=\frac{x^{\mu}}{x^{2}}-b^{\mu}
$$

so a SCT is nothing but the composition of an inversion, a translation characterised by $b^{\mu}$ and again an inversion.

Note that the inversion $\mathbf{x} \rightarrow \mathbf{x} / x^{2}$ satisfies (2.1) and hence is a conformal transformation. But for this transformation to be defined at the origin $x^{\mu}=0$, one should add to space-time an extra point "at infinity" such that the origin is mapped onto it. Moreover, by composition with a translation, any point may be mapped to this point at infinity, and hence it has nothing special: it is an extra space-time point. In two dimensions $d=2$, the resulting space-time $\mathbf{C} \cong \mathbf{R}^{2}$ with this new point "at infinity" has the topology of a sphere and is called the Riemann Sphere. 


\section{Algebra}

Following the general discussion on generators in section 1.2, we easily find the generators of conformal transformations:

$$
\begin{aligned}
P_{\mu} & =-i \partial_{\mu} & & \text { (Translations) } \\
D & =-i x \cdot \partial & & \text { (Dilatations) } \\
L_{\mu \nu} & =i\left(x_{\mu} \partial_{v}-x_{v} \partial_{\mu}\right) & & \text { (Rotations) } \\
K_{\mu} & =-i\left(2 x_{\mu} x \cdot \partial-x^{2} \partial_{\mu}\right) . & & \text { (SCT) }
\end{aligned}
$$

The generators given in (2.15) are valid for fields invariant under conformal transformations, i.e. for $F=0$ in the notations of section 1.1. We will later on consider the action of these generators on fields transforming non-trivially under conformal transformations, see section 2.2.

The algebra satisfied by these generators is

$$
\begin{aligned}
{\left[D, P_{\mu}\right] } & =-i P_{\mu} \\
{\left[D, K_{\mu}\right] } & =-i K_{\mu} \\
{\left[K_{\mu}, P_{v}\right] } & =2 i\left(\delta_{\mu v} D-L_{\mu v}\right) \\
{\left[K_{\rho}, L_{\mu v}\right] } & =i\left(\delta_{\rho \mu} K_{v}-\delta_{\rho v} K_{\mu}\right) \\
{\left[P_{\rho}, L_{\mu v}\right] } & =i\left(\delta_{\rho \mu} P_{v}-\delta_{\rho v} P_{\mu}\right) \\
{\left[L_{\mu v}, L_{\rho \lambda}\right] } & =i\left(\delta_{v \rho} L_{\mu \lambda}+\delta_{\mu \lambda} L_{v \rho}-\delta_{\mu \rho} L_{v \lambda}-\delta_{v \lambda} L_{\mu \rho}\right) .
\end{aligned}
$$

It is now manifest that the Euclidean algebra is a subalgebra of the above conformal algebra, since the commutation relations of $P_{\mu}$ and $L_{\mu \nu}$ do not involve any of the generators $D$ or $K_{\mu}$. We now consider redefinitions of the generators that will allow us to identify the conformal group explicitly. Consider the new generators $\left\{J_{a b}\right\}$ with $a, b, \ldots=-1,0,1, \ldots, d$ defined as

$$
\begin{aligned}
J_{\mu v} & =L_{\mu v} \\
J_{-1,0} & =D \\
J_{-1, \mu} & =\frac{1}{2}\left(P_{\mu}-K_{\mu}\right) \\
J_{0, \mu} & =\frac{1}{2}\left(P_{\mu}+K_{\mu}\right) .
\end{aligned}
$$

We also define $\left(\tilde{\eta}_{a b}\right)$ as the $(d+2) \times(d+2)$ diagonal matrix with

$$
\begin{aligned}
\tilde{\eta}_{-1,-1} & =-1, \\
\tilde{\eta}_{00} & =1, \\
\tilde{\eta}_{\mu v} & =\delta_{\mu v} .
\end{aligned}
$$

Remark that the matrix $\tilde{\eta}$ is of Minkowski signature $(-1,+1, \ldots,+1)$ when we consider Euclidean space-time, while for Minkowskian space-time $\tilde{\eta}$ is of signature $(-1,-1,+1, \ldots,+1)$.

In term of $\tilde{\eta}$ and $J_{a b}$, the conformal algebra (2.16) reads

$$
\left[J_{a b}, J_{c d}\right]=i\left(\tilde{\eta}_{a d} J_{b c}+\tilde{\eta}_{b c} J_{a d}-\tilde{\eta}_{a c} J_{b d}-\tilde{\eta}_{b d} J_{a c}\right) .
$$


We thus conclude that the conformal group in Euclidean space is $S O(d+1,1)$, while in Lorentzian space-time it is $S O(d, 2)$. In either case, a generic conformal transformation has $(d+1)(d+2) / 2$ parameters.

\subsection{Action of conformal transformations on fields}

We now turn to the question of how the fields transform under a general conformal transformation, that is, we look for representations of the conformal group.

Consider a field $\Phi$ of spin $s$. The Poincaré generators act as

$$
\begin{aligned}
P_{\mu} \Phi(\mathbf{x}) & =-i \partial_{\mu} \Phi(\mathbf{x}) \\
L_{\mu \nu} \Phi(\mathbf{x}) & =i\left(x_{\mu} \partial_{\nu}-x_{v} \partial_{\mu}\right) \Phi(\mathbf{x})+S_{\mu v} \Phi(\mathbf{x}),
\end{aligned}
$$

where $S_{\mu \nu}$ are the spin-s representation matrices of the rotation group. Consider first the subgroup of conformal transformations that leave the point $\mathbf{x}=0$ invariant, that is, the subgroup generated by dilatations, rotations and SCTs. The matrices representing the action of these transformations on $\Phi$ are denoted respectively by $\tilde{D}, S_{\mu \nu}$ and $\tilde{K}$. These generators must satisfy

$$
\begin{aligned}
{\left[\tilde{D}, S_{\mu v}\right] } & =0 \\
{\left[\tilde{K}_{\mu}, \tilde{K}_{v}\right] } & =0 \\
{\left[\tilde{D}, \tilde{K}_{\mu}\right] } & =-i \tilde{K}_{\mu} \\
{\left[\tilde{K}_{\rho}, S_{\mu \nu}\right] } & =i\left(\delta_{\rho \mu} \tilde{K}_{v}-\delta_{\rho v} \tilde{K}_{\mu}\right) \\
{\left[S_{\mu \nu}, S_{\rho \lambda}\right] } & =i\left(\delta_{v \rho} S_{\mu \lambda}+\delta_{\mu \lambda} S_{v \rho}-\delta_{\mu \rho} S_{v \lambda}-\delta_{v \lambda} S_{\mu \rho}\right)
\end{aligned}
$$

The action of the generators at any space-time point $\mathbf{x}$ is obtained by conjugation with the translation operator $e^{i \mathbf{x} \cdot P}$. The result may be easily derived using the Hausdorff formula:

$$
e^{-A} B e^{A}=B+[B, A]+\frac{1}{2}[[B, A], A]+\frac{1}{3 !}[[[B, A], A], A]+\cdots
$$

Explicitly,

$$
\begin{aligned}
\tilde{K} \Phi(\mathbf{x}) & =0 \\
D \Phi(\mathbf{x}) & =(-i \mathbf{x} \cdot \partial+\tilde{D}) \Phi(\mathbf{x}) \\
K_{\mu} \Phi(\mathbf{x}) & =\left(\tilde{K}_{\mu}+2 x_{\mu} \tilde{D}-x^{v} S_{\mu \nu}-2 i x_{\mu} x \cdot \partial+i x^{2} \partial_{\mu}\right) \Phi(\mathbf{x})
\end{aligned}
$$

We now restrict our attention to a field $\Phi(\mathbf{x})$ that belongs to an irreducible representation of the rotation group. Since $\tilde{D}$ commutes with all the rotation generators $S_{\mu \nu}, \tilde{D}$ must be proportional to the identity matrix by Schur's lemma. By comparing the action of $\tilde{D}$ on $\Phi(\mathbf{x})$ with the definition of scaling dimension given in section 1 , we deduce that $\tilde{D}=-i \Delta$.

To conclude, let us write the finite transformation for a spinless field $\phi(\mathbf{x})$ under a general conformal transformation: $\phi \rightarrow \phi^{\prime}$ with

$$
\phi^{\prime}\left(\mathbf{x}^{\prime}\right)=\left|\frac{\partial \mathbf{x}^{\prime}}{\partial \mathbf{x}}\right|^{-\Delta / d} \phi(\mathbf{x}) .
$$

Fields transforming this way are called quasi-primary fields. 


\subsection{Energy-momentum tensor and conformal invariance}

Using the definition of energy-momentum tensor (1.48), we have that under a conformal transformation,

$$
\delta S=\frac{1}{d} \int \mathrm{d}^{d} x T_{\mu}^{\mu} \partial \cdot \varepsilon .
$$

Hence if the stress tensor is traceless, then the theory is conformal. The converse, however, is not true in general, for the function $\partial \cdot \varepsilon$ is not arbitrary as we have seen in section 2.1.

Under certain circumstances it is possible to find improvement terms that render the stress tensor traceless. In order to do that we need the theory to be rotation and scale invariant, but as we will see, this may not be enough.

Let us define the virial $V^{\mu}$ of a field $\Phi$ as the combination

$$
V^{\mu}=\frac{\partial L}{\partial\left(\partial^{\rho} \Phi\right)}\left(\delta^{\mu \rho} \Delta+i S^{\mu \rho}\right) \Phi
$$

Then if $V^{\mu}$ can be written as $\partial_{\lambda} \sigma^{\lambda \mu}$ for some tensor $\sigma$, we may construct an improvement term such that the resulting modified energy-momentum tensor is traceless, and hence the theory is conformal. Explicitly, if we define $X^{\lambda \rho \mu v}$ by

$$
\begin{aligned}
X^{\lambda \rho \mu v}=\frac{2}{d-2}\left[\delta^{\lambda \rho} \sigma^{(\mu \nu)}-\delta^{\lambda \mu} \sigma^{(\rho v)}-\delta^{\lambda \mu} \sigma^{(v \rho)}+\delta^{\mu v} \sigma^{(\lambda \rho)}\right. & \\
& \left.+\frac{1}{d-1}\left(\delta^{\lambda \rho} \delta^{\mu v}-\delta^{\lambda \mu} \delta^{\rho v}\right) \sigma^{(\kappa \sigma)} \delta_{\kappa \sigma}\right],
\end{aligned}
$$

then the modified stress tensor $T_{m}{ }^{\mu v}$ is given by

$$
T_{m}{ }^{\mu v}=T_{c}^{\mu v}+\partial_{\rho} B^{\rho \mu v}+\frac{1}{2} \partial_{\lambda} \partial_{\rho} X^{\lambda \rho \mu v},
$$

where $B$ is defined in (1.41). Note that since $X^{(\mu v)[\rho \lambda]}=0, T_{m}{ }^{\mu v}$ is also symmetric and satisfies

$$
\partial_{\mu} j_{D}^{\mu}=T_{m}^{\mu}{ }_{\mu} .
$$

We then see explicitly that in this case, scale invariance implies conformal invariance. Moreover, we get a new expression for the dilatation current, valid when the equations of motion are satisfied:

$$
j_{D}^{\mu}=T_{m}{ }^{\mu}{ }_{v} x^{v}
$$

Let us mention that for two-dimensional quantum field theory and under some broad hypotheses (including unitarity), rotation and scale invariance imply conformal invariance [9].

\subsection{Conformal invariance in quantum field theory and Ward identities}

Let us now turn to the aspects of a quantum conformal field theory. As reviewed in section 1 , all information about a continuous symmetry is encoded in the Ward identities, so let us derive them for conformal transformations. As a warm up, we start by writing them for rotation and translation invariance. 
Consider the canonical current associated to rotational invariance $j^{\mu v \rho}$. Assuming the stress tensor $T^{\mu v}$ has been symmetrized, we have

$$
j^{\mu v \rho}=T^{\mu v} x^{\rho}-T^{\mu \rho} x^{v} .
$$

The corresponding Ward identity is

$$
\begin{aligned}
\partial_{\mu}\left\langle\left(T^{\mu v} x^{\rho}-T^{\mu \rho} x^{v}\right) \Phi_{1}\left(\mathbf{x}_{1}\right)\right. & \left.\cdots \Phi_{n}\left(\mathbf{x}_{n}\right)\right\rangle= \\
& \sum_{i=1}^{n} \delta^{d}\left(\mathbf{x}-\mathbf{x}_{i}\right)\left[x_{i}^{v} \partial_{i}^{\rho}-x_{i}^{\rho} \partial_{i}^{v}-i S_{i}^{v \rho}\right]\left\langle\Phi_{1}\left(\mathbf{x}_{1}\right) \cdots \Phi_{n}\left(\mathbf{x}_{n}\right)\right\rangle,
\end{aligned}
$$

where $\partial_{i}^{\mu}$ denote the derivative with respect to $x_{i}^{\mu}$ and $S_{i}^{\mu v}$ are the generators of the Lorentz group in the representation of the field $\Phi_{i}$. For translations, the Ward identity reads

$$
\partial_{\mu}\left\langle T^{\mu v}(\mathbf{x}) \Phi_{1}\left(\mathbf{x}_{1}\right) \cdots \Phi_{n}\left(\mathbf{x}_{n}\right)\right\rangle=-\sum_{i=1}^{n} \delta^{d}\left(\mathbf{x}-\mathbf{x}_{i}\right) \partial_{i}^{v}\left\langle\Phi_{1}\left(\mathbf{x}_{1}\right) \cdots \Phi_{n}\left(\mathbf{x}_{n}\right)\right\rangle .
$$

Combining equations (2.35) and (2.36) we find

$$
\left\langle\left(T^{\mu v}(\mathbf{x})-T^{v \mu}(\mathbf{x})\right) \Phi_{1}\left(\mathbf{x}_{1}\right) \cdots \Phi_{n}\left(\mathbf{x}_{n}\right)\right\rangle=-i \sum_{i=1}^{n} \delta^{d}\left(\mathbf{x}-\mathbf{x}_{i}\right) S_{i}^{\mu v}\left\langle\Phi_{1}\left(\mathbf{x}_{1}\right) \cdots \Phi_{n}\left(\mathbf{x}_{n}\right)\right\rangle,
$$

which is the quantum analog of the statement that the stress tensor is symmetric: as usual when there are no anomalies for the considered classical invariances, the classical property of symmetry $T^{\mu v}=T^{v \mu}$ translates into a similar statement in terms of correlation functions up to the addition of contact terms, as can be seen on the right hand side of (2.37). If we further assume that the stress tensor is traceless, then the dilatation current $j_{D}^{\mu}$ is

$$
j_{D}^{\mu}=T^{\mu}{ }_{v} x^{v}
$$

yielding the following Ward identity,

$$
\partial_{\mu}\left\langle T^{\mu}{ }_{\nu} x^{v} \Phi_{1}\left(\mathbf{x}_{1}\right) \cdots \Phi_{n}\left(\mathbf{x}_{n}\right)\right\rangle=i \sum_{i=1}^{n} \delta^{d}\left(\mathbf{x}-\mathbf{x}_{i}\right)\left(\mathbf{x}_{i} \cdot \partial_{i}+\Delta_{i}\right)\left\langle\Phi_{1}\left(\mathbf{x}_{1}\right) \cdots \Phi_{n}\left(\mathbf{x}_{n}\right)\right\rangle,
$$

where $\Delta_{i}$ is the scaling dimension of the field $\Phi_{i}$. Equation (2.39) then implies

$$
\left\langle T_{\mu}^{\mu}(\mathbf{x}) \Phi\left(\mathbf{x}_{1}\right) \cdots \Phi\left(\mathbf{x}_{n}\right)\right\rangle=-i \sum_{i=1}^{n} \delta^{d}\left(\mathbf{x}-\mathbf{x}_{i}\right) \Delta_{i}\left\langle\Phi\left(\mathbf{x}_{1}\right) \cdots \Phi\left(\mathbf{x}_{n}\right)\right\rangle
$$

which is the quantum analog of the classical statement that the stress tensor is traceless.

\section{Constraints on two- and three-point functions}

We now consider consequences of conformal invariance on two- and three-point functions, and show that they turn out to be completely fixed, up to global multiplicative constants. Consider the two-point function

$$
\left\langle\phi_{1}\left(\mathbf{x}_{1}\right) \phi_{2}\left(\mathbf{x}_{2}\right)\right\rangle
$$


where $\phi_{1}$ and $\phi_{2}$ are scalar operators that we assume to be quasi-primary and not necessarily appearing in the collection of fields $\left\{\Phi_{i}\right\}$. Under a conformal transformation $\mathbf{x} \mapsto \mathbf{x}^{\prime}$, the quantum invariance condition (1.50) together with (2.27) imply

$$
\left\langle\phi_{1}\left(\mathbf{x}_{1}\right) \phi_{2}\left(\mathbf{x}_{2}\right)\right\rangle=\left|\frac{\partial \mathbf{x}^{\prime}}{\partial \mathbf{x}}\right|_{x_{1}}^{\Delta_{1} / d}\left|\frac{\partial \mathbf{x}^{\prime}}{\partial \mathbf{x}}\right|_{x_{2}}^{\Delta_{2} / d}\left\langle\phi_{1}\left(\mathbf{x}_{1}^{\prime}\right) \phi_{2}\left(\mathbf{x}_{2}^{\prime}\right)\right\rangle .
$$

From rotation and translation invariance, we find that

$$
\left\langle\phi_{1}\left(\mathbf{x}_{1}\right) \phi_{2}\left(\mathbf{x}_{2}\right)\right\rangle=f\left(\left|\mathbf{x}_{1}-\mathbf{x}_{2}\right|\right),
$$

for some function $f$ of one real variable. Focusing on a dilatation $\mathbf{x} \mapsto \lambda \mathbf{x}$ in formula (2.42), we find that the function $f$ defined in (2.43) must be such that

$$
f(s)=\lambda^{\Delta_{1}+\Delta_{2}} f(\lambda s)
$$

We now consider constraint (2.44) at the special point $s=1 / \lambda$. This yields the following formula for $f$,

$$
f(s)=\frac{C_{12}}{s^{\Delta_{1}+\Delta_{2}}},
$$

where $C_{12} \equiv f(1)$ is an unknown constant. Plugging (2.45) into formula (2.43) yields

$$
\left\langle\phi_{1}\left(\mathbf{x}_{1}\right) \phi_{2}\left(\mathbf{x}_{2}\right)\right\rangle=\frac{C_{12}}{\left|\mathbf{x}_{1}-\mathbf{x}_{2}\right|^{\Delta_{1}+\Delta_{2}}} .
$$

If we consider now an SCT defined in (2.13d), it is easy to check that the constraint (2.42) implies that the two-point function (2.41) is automatically zero for $\Delta_{1} \neq \Delta_{2}$. Our final result is therefore

$$
\left\langle\phi_{1}\left(\mathbf{x}_{1}\right) \phi_{2}\left(\mathbf{x}_{2}\right)\right\rangle=\frac{C_{12}}{\left|\mathbf{x}_{1}-\mathbf{x}_{2}\right|^{2 \Delta_{1}}} \quad \text { if } \Delta_{1}=\Delta_{2} \text { and } 0 \text { otherwise. }
$$

In particular, a one-point function (obtained from (2.41) by taking one of the two operators to be the identity) is non-zero only if the scaling dimension of the operator is zero,

$$
\left\langle\phi_{i}(\mathbf{x})\right\rangle \neq 0 \quad \text { iff } \quad \Delta_{i}=0
$$

Let us mention that it is possible to show that in a unitary CFT, identity is the only operator with zero scaling dimension.

Let us now turn to the three-point function,

$$
\left\langle\phi_{1}\left(\mathbf{x}_{1}\right) \phi_{2}\left(\mathbf{x}_{2}\right) \phi_{3}\left(\mathbf{x}_{3}\right)\right\rangle
$$

Using the constraints obtained from (1.50) applied to translations, rotations and dilatations, we find that the three-point function (2.49) must take the form

$$
\left\langle\phi_{1}\left(\mathbf{x}_{1}\right) \phi_{2}\left(\mathbf{x}_{2}\right) \phi_{3}\left(\mathbf{x}_{3}\right)\right\rangle=\frac{C_{123}^{(a b c)}}{x_{12}^{a} x_{23}^{b} x_{13}^{c}}+\text { all permutations in }(a, b, c),
$$


where $C_{123}^{(a b c)}$ are unknown numbers, the three numbers $a, b, c$ satisfy the constraint $a+b+c=\Delta_{1}+$ $\Delta_{2}+\Delta_{3}$ and $x_{i j} \equiv\left|x_{i}-x_{j}\right|$. Applying now to (2.49) the constraint (1.50) for an SCT characterised by a vector $b_{\mu}$ as in (2.13d) yields

$$
\begin{aligned}
& \left\langle\phi_{1}\left(\mathbf{x}_{1}\right) \phi_{2}\left(\mathbf{x}_{2}\right) \phi_{3}\left(\mathbf{x}_{3}\right)\right\rangle=\frac{C_{123}^{(a b c)}}{\gamma_{1}^{\lambda_{1}} \gamma_{2}^{\lambda^{2}} \gamma_{3}^{\Lambda_{3}}} \frac{\left(\gamma_{1} \gamma_{2}\right)^{a / 2}\left(\gamma_{2} \gamma_{3}\right)^{b / 2}\left(\gamma_{1} \gamma_{3}\right)^{c / 2}}{x_{12}^{a} x_{23}^{b} x_{13}^{c}} \\
& + \text { all permutations in }(a, b, c) \text {, }
\end{aligned}
$$

where we defined the quantities $\gamma_{i}$ by

$$
\gamma_{i}=1-2 b_{\mu} x_{i}^{\mu}+b^{2} x_{i}^{2}
$$

Compatibility of the two formula (2.50) and (2.51) requires

$$
a+c=2 \Delta_{1}, \quad a+b=2 \Delta_{2}, \quad b+c=2 \Delta_{3} .
$$

Solving the system (2.53) for $a, b, c$ in terms of $\Delta_{1}, \Delta_{2}$ and $\Delta_{3}$, we find that

$$
\left\langle\phi_{1}\left(\mathbf{x}_{1}\right) \phi_{2}\left(\mathbf{x}_{2}\right) \phi_{3}\left(\mathbf{x}_{3}\right)\right\rangle=\frac{C_{123}}{x_{12}^{\Delta_{1}+\Delta_{2}-\Delta_{3}} x_{23}^{\Delta_{2}+\Delta_{3}-\Delta_{1}} x_{13}^{\Delta_{3}+\Delta_{1}-\Delta_{2}}},
$$

where $C_{123}$ is an undetermined coefficient.

\section{Comment about the non-existence of an S-matrix in non-trivial CFTs}

As a consequence of scale invariance, there is no physically meaningful notion of distance in a conformal field theory. It is then impossible to define an S-matrix in a standard fashion, where we assume that interactions are negligible when wave packets are separated enough. Another way to see this is by remembering that inversions are now symmetries of the theory, and if interactions are assumed to tend to zero at infinity, then they should be zero everywhere. The concept of observables cannot be defined in a standard fashion; for our purposes, we shall assume that correlators are the physically relevant quantities to consider.

\section{Conformal invariance in two dimensions}

We now turn to the study of conformal field theories in two dimensions. Actually, much of what we derived in section 2 remains valid when $d=2$, because the set of conformal transformations in two dimensions contains those satisfying (2.9). As we shall see, there are however much more solutions to the constraints (2.1) in two dimensions.

\subsection{Identification of the transformations and analysis}

Let us slightly change our notations and call $\left(z^{0}, z^{1}\right)$ our space-time coordinates. Recall that the definition of a conformal transformation $z^{\mu} \mapsto w^{\mu}(z)$ is

$$
\delta^{\rho \lambda}=\frac{\partial w^{\rho}}{\partial z^{\mu}} \frac{\partial w^{\lambda}}{\partial z^{v}} \delta^{\mu v}
$$


Explicitely, this is equivalent to

$$
\begin{aligned}
\left(\frac{\partial w^{0}}{\partial z^{0}}\right)^{2}+\left(\frac{\partial w^{0}}{\partial z^{1}}\right) & =\left(\frac{\partial w^{1}}{\partial z^{0}}\right)^{2}+\left(\frac{\partial w^{1}}{\partial z^{1}}\right)^{2}, \\
\frac{\partial w^{0}}{\partial z^{0}} \frac{\partial w^{1}}{\partial z^{0}}+\frac{\partial w^{0}}{\partial z^{1}} \frac{\partial w^{1}}{\partial z^{1}} & =0
\end{aligned}
$$

There are two ways one can solve these equations for $w^{\mu}(z)$ : either we set

$$
\frac{\partial w^{0}}{\partial z^{0}}=-\frac{\partial w^{1}}{\partial z^{1}}, \quad \frac{\partial w^{0}}{\partial z^{1}}=\frac{\partial w^{1}}{\partial z^{0}}
$$

or we can choose

$$
\frac{\partial w^{0}}{\partial z^{0}}=\frac{\partial w^{1}}{\partial z^{1}}, \quad \frac{\partial w^{0}}{\partial z^{1}}=-\frac{\partial w^{1}}{\partial z^{0}}
$$

These a precisely the Cauchy-Riemann relations for (respectively) an holomorphic or an antiholomorphic function! It is then very convenient to define the complex variables $z=z^{0}+i z^{1}$ and $\bar{z}=z^{0}-i z^{1}$ and extend space-time to a complex space-time, where $\bar{z}$ is considered as independent of $z$. We recover our original space-time by imposing the reality condition $z^{*}=\bar{z}$. We have the usual complex analysis relations

$$
\begin{aligned}
z^{0} & =\frac{1}{2}(z+\bar{z}), & z^{1} & =\frac{1}{2 i}(z-\bar{z}), \\
\partial_{z} & =\frac{1}{2}\left(\partial_{0}-i \partial_{1}\right), & \partial_{\bar{z}} & =\frac{1}{2}\left(\partial_{0}+i \partial_{1}\right), \\
\partial_{0} & =\partial_{z}+\partial_{\bar{z}}, & \partial_{1} & =i\left(\partial_{z}-\partial_{\bar{z}}\right) .
\end{aligned}
$$

In these coordinates, the metric $\delta=\left(\delta_{\mu v}\right)$ and its inverse $\delta^{-1}$ read

$$
\delta=\left(\begin{array}{cc}
0 & 1 / 2 \\
1 / 2 & 0
\end{array}\right), \quad \delta^{-1}=\left(\begin{array}{ll}
0 & 2 \\
2 & 0
\end{array}\right)
$$

We also define the antisymmetric tensor $\left(\varepsilon_{\mu \nu}\right)$ by

$$
\varepsilon=\left(\begin{array}{cc}
0 & i / 2 \\
-i / 2 & 0
\end{array}\right), \quad \varepsilon^{-1}=\left(\begin{array}{cc}
0 & -2 i \\
2 i & 0
\end{array}\right)
$$

Note that we did not yet imposed that the coordinate transformation is everywhere well defined, and the Cauchy-Riemann relations are of course valid only when evaluated at points where the functions $(w(z), \bar{w}(\bar{z}))$ exist. In the rest of these notes, when we say that " $f$ is holomorphic" (or "anti-holomorphic") we actually mean meromorphic (or anti-meromorphic), that is, holomorphic (or anti-holomorphic) on the whole complexified space-time except maybe at some isolated points where the function may have poles, and we write $f(z)$ (or $f(\bar{z})$ respectively). The set of conformal transformations in two dimensions is hence infinite-dimensional, as it consists of all holomorphic and anti-holomorphic maps of the Riemann sphere. 


\section{Generators}

We consider an infinitesimal transformation $z \mapsto z+\varepsilon(z), \bar{z} \mapsto \bar{z}+\bar{\varepsilon}(\bar{z})$, where $\varepsilon$ is holomorphic and $\bar{\varepsilon}$ is antiholomoprhic. We assume that $\varepsilon$ admits, at least in a non-empty open set, the following Laurent series around zero:

$$
\varepsilon(z)=\sum_{n=-\infty}^{\infty} c_{n} z^{n+1}
$$

where $c_{n}$ are its Laurent coefficients, and similarly for $\bar{\varepsilon}$ with some coefficient $\bar{c}_{n}$ (that are not a priori related to the coefficients $c_{n}$ ). Consider a scalar field $\phi=\phi(z, \bar{z})$. Since we are in two dimensions, the scaling dimension of $\phi$ is $\Delta=0$, and hence under our infinitesimal conformal transformation we have

$$
\delta \phi(z, \bar{z})=\phi^{\prime}(z, \bar{z})-\phi(z, \bar{z})=-\partial_{z} \phi(z, \bar{z}) \varepsilon(z)-\partial_{\bar{z}} \phi(z, \bar{z}) \bar{\varepsilon}(\bar{z}) .
$$

The generators $\ell_{n}, \bar{\ell}_{n}$ are defined so that

$$
\delta \phi=\sum_{n=-\infty}^{\infty}\left(c_{n} \ell_{n} \phi+\bar{c}_{n} \bar{\ell}_{n} \phi\right)
$$

and hence

$$
\ell_{n}=-z^{n+1} \partial_{z}, \quad \bar{\ell}_{n}=-\bar{z}^{n+1} \partial_{\bar{z}}
$$

It is easy to show that they satisfy the following commutation relations:

$$
\begin{aligned}
& {\left[\ell_{n}, \ell_{m}\right]=(n-m) \ell_{n+m},} \\
& {\left[\bar{\ell}_{n}, \bar{\ell}_{m}\right]=(n-m) \bar{\ell}_{n+m},} \\
& {\left[\ell_{n}, \bar{\ell}_{m}\right]=0 .}
\end{aligned}
$$

Note that the two sets of generators $\ell_{n}$ and $\bar{\ell}_{n}$ commute. The algebra defined by the relation (3.13a) is a Witt algebra for the generators $\ell_{n}$ (and similarly for the $\bar{\ell}_{n}$ ) and thus the algebra (3.13) of the generators of conformal transformation in two dimensions at the classical level consists of two copies of the Witt algebra.

\section{Global conformal transformations}

In this section we are interested in conformal transformations that are globally defined on the whole complex Riemann sphere. If such transformations leave the action untouched, then they are called "true symmetries" or "global symmetries," as they are the only ones that provide conserved currents that are everywhere well defined.

Given the form (3.12) for the generators, we see that only $\ell_{n}$ with $n \geq-1$ are defined at the origin. Moreover, one should require them to be also well defined at the point at infinity, since the latter belongs to the Riemann sphere. Setting $w=1 / z$, we find that $\ell_{n}=w^{1-n} \partial_{w}$, which is well defined at $w=0$ only for $n \leq 1$ (similar conclusions apply for $\bar{\ell}_{n}$ ). Therefore, there are only 6 generators that correspond to conformal transformations that are well defined on the complex Riemann sphere:

$$
\left\{\ell_{0}, \ell_{1}, \ell_{-1}\right\} \quad \text { and } \quad\left\{\bar{\ell}_{0}, \bar{\ell}_{1}, \bar{\ell}_{-1}\right\}
$$


As a consequence of (3.13), these generators satisfy the following algebra:

$$
\begin{aligned}
{\left[\ell_{0}, \ell_{-1}\right] } & =\ell_{-1}, \\
{\left[\ell_{1}, \ell_{0}\right] } & =\ell_{1}, \\
{\left[\ell_{1}, \ell_{-1}\right] } & =2 \ell_{0} .
\end{aligned}
$$

and similarly for $\bar{\ell}_{n}$, with $n=-1,0,1$. Algebra (3.15) is a subalgebra of the Witt algebra (3.13a). Here are the infinitesimal and finite transformations corresponding to these generators (since the algebra for the $\bar{\ell}_{n}$ is similar to the one for the $\ell_{n}$, we focus on the holomorphic part and similar results will apply for the anti-holomorphic part):

$$
\begin{array}{rll}
\ell_{-1}: & z \mapsto z+\varepsilon, & z \mapsto z+\alpha, \\
\ell_{0}: & z \mapsto z+\varepsilon z, & z \mapsto \lambda z, \\
\ell_{1}: & z \mapsto z+\varepsilon z^{2}, \quad z \mapsto \frac{z}{1-\beta z},
\end{array}
$$

where $\varepsilon, \alpha, \lambda$ and $\beta$ are complex parameters, $\varepsilon$ being in addition infinitesimal. Combining the three above transformations, we find that a general global holomorphic conformal transformation is of the form

$$
z \rightarrow \frac{a z+b}{c z+d}
$$

where the complex numbers $a, b, c, d$ are such that $a d-b c=1$. It is easy to check that the result of the composition of two such transformations (parametrized, say, by $a_{1}, b_{1}, c_{1}, d_{1}$ and $a_{2}, b_{2}, c_{2}, d_{2}$ ) corresponds to a transformation parametrized by $a, b, c, d$ with $a d-c b=1$ and such that

$$
\left(\begin{array}{ll}
a & b \\
c & d
\end{array}\right)=\left(\begin{array}{ll}
a_{2} & b_{2} \\
c_{2} & d_{2}
\end{array}\right)\left(\begin{array}{ll}
a_{1} & b_{1} \\
c_{1} & d_{1}
\end{array}\right) .
$$

Global holomorphic conformal transformations hence form a group isomorphic to $\operatorname{SL}(2, \mathbf{C}) / \mathbf{Z}_{2}$ with matrix multiplication, the $\mathbf{Z}_{2}$ factor coming from the fact that flipping the sign of $a, b, c, d$ returns the same conformal transformation (3.17) and preserves the relation $a d-b c=1$.

Similar results apply for anti-holomorphic global conformal transformations. If we reduce to real space-time by imposing $\bar{z}=z^{*}$, it can be shown that the resulting group for global conformal transformations is $S O(3,1)$. Recall that for $d \geq 3$, the conformal group is $S O(d+1,1)$, and therefore we have shown that this result is actually also valid for $d=2$. This means that all results derived in section 2 from invariance under $S O(d+1,1)$, as for instance the transformation law for scalar fields, Ward identities and constraints on two- and three-point functions apply for $d=2$ as well.

\section{Transformation laws for fields with arbitrary spin and primary fields}

Consider a field $\Phi$ of spin $s$ and scaling dimension $\Delta$. We define for this field its holomorphic weight $h$ and anti-holomorphic weight $\bar{h}$ by

$$
h=\frac{\Delta+s}{2}, \quad \bar{h}=\frac{\Delta-s}{2},
$$


and consider a global conformal transformation $z \rightarrow w(z), \bar{z} \rightarrow \bar{w}(\bar{z})$. Then it can be shown ${ }^{3}$ that the formula (2.27), valid for scalar fields only, generalizes to

$$
\Phi^{\prime}(w, \bar{w})=\left(\frac{\mathrm{d} w}{\mathrm{~d} z}\right)^{-h}\left(\frac{\mathrm{d} \bar{w}}{\mathrm{~d} \bar{z}}\right)^{-\bar{h}} \Phi(z, \bar{z}) .
$$

Again, fields transforming this way are termed quasi-primary fields. If, in addition, the field $\Phi$ transforms as (3.20) for any conformal transformation (not necessarily global), then $\Phi$ is called a primary field. Obviously, a primary field is always quasi-primary, but the converse is not true. As we will shortly see, the energy-momentum tensor of a generic CFT is an example of quasi-primary that is not primary.

For future reference, we note that under a purely holomorphic conformal transformation $z \mapsto$ $z+\varepsilon(z)$, we have for a primary field $\Phi$ that $\Phi \mapsto \Phi+\delta_{\varepsilon} \Phi$ with

$$
\delta_{\varepsilon} \Phi=-\varepsilon \partial \Phi-h \partial \varepsilon
$$

where $\partial$ is a shorthand for $\partial_{z}$. We also write $\bar{\partial}=\partial_{\bar{z}}$ in the following.

\subsection{Ward identities}

We will now write the Ward identities for translation, rotation and scale invariance in two dimensions. Note that the generators of rotations $S_{\mu \nu}$, being two by two antisymmetric matrices, are automatically proportional to $\varepsilon_{\mu \nu}$. The proportionality factor is nothing but the spin $s$ of the representation: $S_{\mu v}=s \varepsilon_{\mu v}$. With this observation, the Ward identities (2.36), (2.37) and (2.40) are (in any coordinates) equivalent to

$$
\begin{aligned}
\partial_{\mu}\left\langle T^{\mu v}(\mathbf{x}) \Phi_{1}\left(\mathbf{x}_{1}\right) \cdots \Phi_{n}\left(\mathbf{x}_{n}\right)\right\rangle & =-\sum_{i=1}^{n} \delta^{2}\left(\mathbf{x}-\mathbf{x}_{i}\right) \partial_{i}^{v}\left\langle\Phi_{1}\left(\mathbf{x}_{1}\right) \cdots \Phi_{n}\left(\mathbf{x}_{n}\right)\right\rangle \\
\varepsilon_{\mu \nu}\left\langle T^{\mu v}(\mathbf{x}) \Phi_{1}\left(\mathbf{x}_{1}\right) \cdots \Phi_{n}\left(\mathbf{x}_{n}\right)\right\rangle & =-i \sum_{i=1}^{n} \delta^{2}\left(\mathbf{x}-\mathbf{x}_{i}\right) s_{i}\left\langle\Phi_{1}\left(\mathbf{x}_{1}\right) \cdots \Phi_{n}\left(\mathbf{x}_{n}\right)\right\rangle \\
\left\langle T^{\mu}(\mathbf{x}) \Phi_{1}\left(\mathbf{x}_{1}\right) \cdots \Phi_{n}\left(\mathbf{x}_{n}\right)\right\rangle & =-\sum_{i=1}^{n} \delta^{2}\left(\mathbf{x}-\mathbf{x}_{i}\right) \Delta_{i}\left\langle\Phi_{1}\left(\mathbf{x}_{1}\right) \cdots \Phi_{n}\left(\mathbf{x}_{n}\right)\right\rangle
\end{aligned}
$$

We now specialize to our complex coordinates $(z, \bar{z})$ defined in (3.6). Using the standard formula

$$
\delta^{2}(\mathbf{x})=\frac{1}{\pi} \bar{\partial} \frac{1}{z}=\frac{1}{\pi} \partial \frac{1}{\bar{z}}
$$

\footnotetext{
${ }^{3}$ To convince yourself, you might want to start by showing this formula for an infinitesimal transformation.
} 
we find the formulas

$$
\begin{array}{r}
2 \pi \partial_{z}\left\langle T_{\bar{z} z} \Phi_{1}\left(w_{1}, \bar{w}_{1}\right) \cdots \Phi_{n}\left(w_{n}, \bar{w}_{n}\right)\right\rangle+2 \pi \partial_{\bar{z}}\left\langle T_{z z} \Phi_{1}\left(w_{1}, \bar{w}_{1}\right) \cdots \Phi_{n}\left(w_{n}, \bar{w}_{n}\right)\right\rangle \\
=-\sum_{i=1}^{n} \partial_{\bar{z}} \frac{1}{z-w_{i}} \partial_{w_{i}}\left\langle\Phi_{1}\left(w_{1}, \bar{w}_{1}\right) \cdots \Phi_{n}\left(w_{n}, \bar{w}_{n}\right)\right\rangle \\
2 \pi \partial_{z}\left\langle T_{\bar{z} \bar{z}} \Phi_{1}\left(w_{1}, \bar{w}_{1}\right) \cdots \Phi_{n}\left(w_{n}, \bar{w}_{n}\right)\right\rangle+2 \pi \partial_{\bar{z}}\left\langle T_{z \bar{z}} \Phi_{1}\left(w_{1}, \bar{w}_{1}\right) \cdots \Phi_{n}\left(w_{n}, \bar{w}_{n}\right)\right\rangle \\
=-\sum_{i=1}^{n} \partial_{z} \frac{1}{\bar{z}-\bar{w}_{i}} \partial_{\bar{w}_{i}}\left\langle\Phi_{1}\left(w_{1}, \bar{w}_{1}\right) \cdots \Phi_{n}\left(w_{n}, \bar{w}_{n}\right)\right\rangle \\
2\left\langle T_{z \bar{z}} \Phi_{1}\left(w_{1}, \bar{w}_{1}\right) \cdots \Phi_{n}\left(w_{n}, \bar{w}_{n}\right)\right\rangle+2\left\langle T_{\bar{z} z} \Phi_{1}\left(z_{1}, \bar{z}_{1}\right) \cdots \Phi_{n}\left(z_{n}, \bar{z}_{n}\right)\right\rangle \\
=-\sum_{i=1}^{n} \delta^{2}\left(z-w_{i}\right) \Delta_{i}\left\langle\Phi_{1}\left(w_{1}, \bar{w}_{1}\right) \cdots \Phi_{n}\left(w_{n}, \bar{w}_{n}\right)\right\rangle \\
-2\left\langle T_{z \bar{z}} \Phi_{1}\left(w_{1}, \bar{w}_{1}\right) \cdots \Phi_{n}\left(w_{n}, \bar{w}_{n}\right)\right\rangle+2\left\langle T_{\bar{z} z} \Phi_{1}\left(z_{1}, \bar{z}_{1}\right) \cdots \Phi_{n}\left(z_{n}, \bar{z}_{n}\right)\right\rangle \\
=-\sum_{i=1}^{n} \delta^{2}\left(z-w_{i}\right) s_{i}\left\langle\Phi_{1}\left(w_{1}, \bar{w}_{1}\right) \cdots \Phi_{n}\left(w_{n}, \bar{w}_{n}\right)\right\rangle
\end{array}
$$

Combining the formulas (3.24c) and (3.24d) yields

$$
\begin{aligned}
& 2 \pi\left\langle T_{\bar{z} z} \Phi_{1}\left(w_{1}, \bar{w}_{1}\right) \cdots \Phi_{n}\left(w_{n}, \bar{w}_{n}\right)\right\rangle=-\sum_{i=1}^{n} \partial_{\bar{z}} \frac{1}{z-w_{i}} h_{i}\left\langle\Phi_{1}\left(w_{1}, \bar{w}_{1}\right) \cdots \Phi_{n}\left(w_{n}, \bar{w}_{n}\right)\right\rangle, \\
& 2 \pi\left\langle T_{z \bar{z}} \Phi_{1}\left(w_{1}, \bar{w}_{1}\right) \cdots \Phi_{n}\left(w_{n}, \bar{w}_{n}\right)\right\rangle=-\sum_{i=1}^{n} \partial_{z} \frac{1}{\bar{z}-\bar{w}_{i}} \bar{h}_{i}\left\langle\Phi_{1}\left(w_{1}, \bar{w}_{1}\right) \cdots \Phi_{n}\left(w_{n}, \bar{w}_{n}\right)\right\rangle,
\end{aligned}
$$

and therefore, introducing

$$
T=-2 \pi T_{z z}, \quad \bar{T}=-2 \pi T_{\bar{z} \bar{z}},
$$

we finally find

$$
\begin{aligned}
\partial_{\bar{z}}\left[\left\langle T(z, \bar{z}) \Phi_{1}\left(w_{1}, \bar{w}_{1}\right) \cdots \Phi_{n}\left(w_{n}, \bar{w}_{n}\right)\right\rangle\right. & -\sum_{i=1}^{n}\left(\frac{1}{z-w_{i}} \partial_{w_{i}}\left\langle\Phi_{1}\left(w_{1}, \bar{w}_{1}\right) \cdots \Phi_{n}\left(w_{n}, \bar{w}_{n}\right)\right\rangle\right. \\
& \left.\left.+\frac{h_{i}}{\left(z-w_{i}\right)^{2}}\left\langle\Phi_{1}\left(w_{1}, \bar{w}_{1}\right) \cdots \Phi_{n}\left(w_{n}, \bar{w}_{n}\right)\right\rangle\right)\right]=0,
\end{aligned}
$$

with a similar expression for $\bar{T}$. The quantity between brackets is hence holomorphic everywhere, and in our notations we may replace $T(z, \bar{z})$ by $T(z)$. One should not forget, however, that $T(z)$ fails to be holomorphic when inserted at coincident point in a correlation function. Similarly, $\bar{T}(z, \bar{z})$ is replaced by $\bar{T}(\bar{z})$.

\section{Transformation of correlation functions of primaries}

We now derive a formula giving the variation of a correlation function of primaries under an infinitesimal conformal transformation $\mathbf{x} \mapsto \mathbf{x}+\varepsilon(\mathbf{x})$. Using (3.21) with the definition (3.19) for the holomorphic and anti-holomorphic weights, the correlation function $\left\langle\Phi\left(\mathbf{x}_{1}\right) \cdots \Phi\left(\mathbf{x}_{n}\right)\right\rangle$ of primaries $\Phi\left(\mathbf{x}_{1}\right), \ldots, \Phi\left(\mathbf{x}_{n}\right)$ varies according to

$$
\delta_{\varepsilon}\left\langle\Phi_{1}\left(\mathbf{x}_{1}\right) \cdots \Phi_{n}\left(\mathbf{x}_{n}\right)\right\rangle=-\sum_{i=1}^{n}\left(\varepsilon \cdot \partial_{i}+\frac{\partial \cdot \varepsilon}{2} \Delta_{i}+\frac{\varepsilon^{\mu v} \partial_{\mu} \varepsilon_{v}}{2} s_{i}\right)\left\langle\Phi_{1}\left(\mathbf{x}_{1}\right) \cdots \Phi_{n}\left(\mathbf{x}_{n}\right)\right\rangle .
$$


On the other hand let us consider the following integral,

$$
\int_{D} \mathrm{~d}^{2} x\left\langle\partial_{\mu}\left(\varepsilon_{v}(\mathbf{x}) T^{\mu v}(\mathbf{x})\right) \Phi_{1}\left(\mathbf{x}_{1}\right) \cdots \Phi_{n}\left(\mathbf{x}_{n}\right)\right\rangle,
$$

where the domain $D$ contains all points $\mathbf{x}_{i}$ at which the operators are inserted. This integral is not zero only because of the singularities of the correlations function at coincident points. Performing the derivative in (3.30) and decomposing the term with $\partial_{\mu} \varepsilon_{v}$ in its symmetric and antisymmetric part, ${ }^{4}$ we find for the integrand of (3.30)

$$
\begin{aligned}
\left\langle\partial_{\mu}\left(\varepsilon_{v}(\mathbf{x}) T^{\mu v}(\mathbf{x})\right)\right. & \left.\Phi_{1}\left(\mathbf{x}_{1}\right) \cdots \Phi_{n}\left(\mathbf{x}_{n}\right)\right\rangle \\
= & -\sum_{i=1}^{n} \delta^{2}\left(\mathbf{x}-\mathbf{x}_{i}\right)\left[\varepsilon \cdot \partial_{i}+\frac{\partial \cdot \varepsilon}{2} \Delta_{i}+\frac{\varepsilon^{\mu v} \partial_{\mu} \varepsilon_{v}}{2} s_{i}\right]\left\langle\Phi_{1}\left(\mathbf{x}_{1}\right) \cdots \Phi_{n}\left(\mathbf{x}_{n}\right)\right\rangle .
\end{aligned}
$$

Thanks to the $\delta$-functions in the right hand side of (3.31), the integral (3.30) is trivial to perform, and (3.29) we end up with

$$
\delta_{\varepsilon}\left\langle\Phi\left(\mathbf{x}_{1}\right) \cdots \Phi\left(\mathbf{x}_{n}\right)\right\rangle=\int_{D} \mathrm{~d}^{2} x \partial_{\mu}\left\langle T^{\mu v}(\mathbf{x}) \varepsilon_{v}(\mathbf{x}) \Phi\left(\mathbf{x}_{1}\right) \cdots \Phi\left(\mathbf{x}_{n}\right)\right\rangle .
$$

We now apply Gauss's theorem and specialize to the complex coordinates defined in (3.6). Using the definitions (3.27) of $T$ and $\bar{T}$ we finally find

$$
\begin{aligned}
\delta_{\varepsilon, \bar{\varepsilon}}\left\langle\Phi\left(w_{1}, \bar{w}_{1}\right) \cdots \Phi\left(w_{n}, \bar{w}_{n}\right)\right\rangle=-\frac{1}{2 \pi i} \oint_{C} \mathrm{~d} z \varepsilon(z)\left\langle T(z) \Phi\left(w_{1}, \bar{w}_{1}\right) \cdots \Phi\left(w_{n}, \bar{w}_{n}\right)\right\rangle+ \\
\frac{1}{2 \pi i} \oint \mathrm{d} \bar{z} \bar{\varepsilon}(\bar{z})\left\langle\bar{T}(\bar{z}) \Phi\left(w_{1}, \bar{w}_{1}\right) \cdots \Phi\left(w_{n}, \bar{w}_{n}\right)\right\rangle,
\end{aligned}
$$

where $C=\partial D$ is the boundary of $D$ and we explicitly wrote that the variation depends on two (independent) functions $\varepsilon$ and $\bar{\varepsilon}$. Using the general formula (1.51) relating the variation of a correlator under a symmetry transformation and the corresponding conserved current, the currents $j(z)$ and $\bar{j}(\bar{z})$ associated with holomorphic and anti-holomorphic transformations respectively are straightforwardly read-off from the Ward identity (3.33):

$$
j(z)=\varepsilon(z) T(z), \quad \bar{j}(\bar{z})=\bar{\varepsilon}(\bar{z}) \bar{T}(\bar{z}) .
$$

While we assumed that the fields $\Phi\left(\mathbf{x}_{i}\right)$ appearing in the equation (3.33) are primaries, it may be shown that the same formula holds for non-primaries. This will be clearer in the operator formalism described in section 3.3.

For primaries, we may actually push further formula (3.33), thanks to equation (3.28) and performing the contour integration using Cauchy's theorem. In the case of a purely holomorphic transformation $z \mapsto z+\varepsilon(z)$, we find

$$
\delta_{\varepsilon}\left\langle\Phi_{1}\left(\mathbf{x}_{1}\right) \cdots \Phi_{n}\left(\mathbf{x}_{n}\right)\right\rangle=-\sum_{i=1}^{n}\left(\varepsilon\left(w_{i}\right) \partial_{w_{i}}+\partial \varepsilon\left(w_{i}\right)\right)\left\langle\Phi_{1}\left(\mathbf{x}_{1}\right) \cdots \Phi_{n}\left(\mathbf{x}_{n}\right)\right\rangle,
$$

and similarly for an anti-holomorphic transformation $\bar{z} \mapsto \bar{z}+\bar{\varepsilon}(\bar{z})$.

\footnotetext{
${ }^{4}$ If your are confused by the presence of an antisymmetric piece, recall that symmetry of $T^{\mu v}$ in a correlation function is violated by contact terms (if any), see (2.37).
} 


\subsection{Operator Formalism}

Quantum field theories are more frequently studied in the path integral formalism, as it is for instance very convenient to establish Feynman rules or describe semi-classical effects from the formal expression of correlators in terms of path integrals weighted by $e^{-S}$. In two-dimensional CFT field theories however, it turns out that the operator formalism, which is equivalent to the path integral formalism, is much more convenient. In particular, transformations of fields and operators are very conveniently described by the Operator Product Expansion that we will introduce shortly. In the operator formalism, a classical theory is quantized by defining a Hilbert space, promoting fields to operators on this Hilbert space and giving their equal-time commutation relations. In particular, a coordinate ("time") is singled out. In Euclidean space-time however, there is no preferred direction for a time-like coordinate. We shall hence choose it arbitrarily, although in other contexts (like string theory for example) this is actually the natural choice: time will be the radial direction in the complex plane. The resulting quantization is called radial quantization.

\subsubsection{Radial order and mode expansion}

Even if this was implicit in all our formulas, one should not forget that there is, in any correlator we considered so far, a time-ordering operator that sorts the fields in decreasing time order from left to right. In radial quantization, time ordering is replaced by radial ordering that we define with the operator $R$ with

$$
R\left[\Phi_{1}(z) \Phi_{2}(w)\right]=\left\{\begin{aligned}
\Phi_{1}(z) \Phi_{2}(w) & \text { if }|z|>|w| \\
\pm \Phi_{2}(w) \Phi_{1}(z) & \text { if }|z|<|w|,
\end{aligned}\right.
$$

where the plus sign is selected if one of the operators $\Phi_{1}$ or $\Phi_{2}$ is a commuting-number valued operator, while we chose the minus sign if both $\Phi_{1}$ and $\Phi_{2}$ are anticommuting-number valued. Although we will not write explicitly the radial ordering operator $R$, it should be kept in mind that it is present in any expression we consider.

Let us consider two arbitrary operators $A$ and $B$, and assume they may be represented as

$$
A=\oint_{o} \mathrm{~d} z a(z), \quad B=\oint_{o} \mathrm{~d} z b(z),
$$

where $a(z)$ and $b(z)$ are some operators depending on $z$ and the integrals are taken over a small circle around the origin of the complex plane. If we denote by $\oint_{C_{w}}$ the integral taken on a small circle around a point $w$ of complex Riemann sphere, we have

$$
\oint_{C_{w}} \mathrm{~d} z a(z) b(w)=\oint_{C_{o}^{+}} \mathrm{d} z a(z) b(w)-\oint_{C_{o}^{-}} \mathrm{d} z b(w) a(z)=[A, b(w)],
$$

where $C_{o}^{+}$is a circle centred at the origin with radius slightly bigger that $|w|, C_{o}^{-}$is a circle also centred at the origin but with radius slightly smaller that $|w|$ and we have used the implicit presence of the radial operator $R$ on the left hand side. We therefore conclude that the commutator $[A, B]$ of the two operators $A$ and $B$ can be expressed, using $a(z)$ and $b(z)$, as

$$
[A, B]=\oint_{C_{o}} \mathrm{~d} w \oint_{C_{w}} \mathrm{~d} z a(z) b(w) .
$$


We thus see that $[A, B]$ essentially depends on the singular values of the product of the operators $a(z)$ with $b(w)$ as $z \rightarrow w$. This observation will be our motivation to define the operator product expansion in subsection 3.3.2.

We introduce the following mode expansion ${ }^{5}$ by decomposing the energy-momentum operators $T$ and $\bar{T}$ as

$$
T(z)=\sum_{n=-\infty}^{\infty} \frac{L_{n}}{z^{n+2}}, \quad \bar{T}(\bar{z})=\sum_{n=-\infty}^{\infty} \frac{\bar{L}_{n}}{\bar{z}^{n+2}},
$$

for some operators $L_{n}, \bar{L}_{m}$ whose interpretation will be given in a moment. These relations may be easily inverted using Cauchy's theorem:

$$
L_{n}=\frac{1}{2 \pi i} \oint_{C_{o}} \mathrm{~d} z z^{n+1} T(z), \quad \bar{L}_{n}=\frac{1}{2 \pi i} \oint_{C_{o}} \mathrm{~d} \bar{z} \bar{z}^{n+1} \bar{T}(\bar{z}) .
$$

We will now show that the operators $L_{n}, \bar{L}_{m}$ are the conserved charges associated to conformal transformations. Let us consider an infinitesimal holomorphic conformal transformation $z \mapsto z+$ $\varepsilon(z)$. In the radial quantization scheme, the associated conserved charge $Q_{\varepsilon}$, defined by (1.34), reads

$$
Q_{\varepsilon}=\frac{1}{2 \pi i} \oint_{C_{o}} \mathrm{~d} z j_{\varepsilon}(z)=\frac{1}{2 \pi i} \oint_{C_{o}} \mathrm{~d} z \varepsilon(z) T(z),
$$

where we used the explicit expression (3.34) for the current $j_{\varepsilon}(z)$. Therefore, the associated variation $\delta_{\varepsilon} \Phi$ reads

$$
\delta_{\varepsilon} \Phi(w)=-\left[Q_{\varepsilon}, \Phi(w)\right]=-\frac{1}{2 \pi i} \oint_{C_{o}} \mathrm{~d} z \varepsilon(z) T(z) \Phi(w) .
$$

We now decompose $\varepsilon$ as

$$
\varepsilon(z)=\sum_{n=-\infty}^{\infty} z^{n+2} \varepsilon_{n}
$$

for some $\varepsilon_{n}$ parameterising the transformation, and we finally find that

$$
Q_{\varepsilon}=\sum_{n=-\infty}^{\infty} \varepsilon_{n} L_{n}
$$

We deduce from (3.45) that the operators $L_{n}$ are the conserved charges corresponding to conformal invariance (for holomorphic transformations). A similar result holds for anti-holomorphic transformations, and so the $L_{n}, \bar{L}_{m}$ are the quantum analogues of $\ell_{n}, \bar{\ell}_{m}$ introduced in (3.11). In order to determine the algebra satisfied by the operators $L_{m}, \bar{L}_{n}$, we first need to introduce the concept of operator product expansion.

\subsubsection{Operator Product Expansion}

We now introduce the concept of operator product expansion, or OPE for short. Let $a(z)$ and $b(w)$ be some operators. Typically, the expectation value $\langle a(z) b(w)\rangle$ diverges as some negative power of $|z-w|$ when $z \rightarrow w$. Moreover, as we have seen in the previous section (see for example formula (3.33)) such expectation values often appear in a contour integral and thus by Cauchy's theorem, the result of such typical integrations will be completely determined by the singular part

\footnotetext{
${ }^{5}$ This terminology is more natural in polar coordinates, but we shall nevertheless use it.
} 
of the integrand. To get some control on this, we assume that the product $a(z) b(w)$ is given by (we restrict to holomorphic operators for convenience)

$$
a(z) b(w)=\sum_{n=-\infty}^{N} \frac{\{a b\}_{n}(w)}{(z-w)^{n}}
$$

for some operators $\{a b\}_{n}$. The Operator Product Expansion is defined as the sum of the singular terms in the series of the right hand side of (3.46), and we write

$$
a(z) b(w) \sim \sum_{n=1}^{N} \frac{\{a b\}_{n}(w)}{(z-w)^{n}},
$$

where by definition the right hand side is the OPE of the left hand side. As an equality between operators, (3.47) should always be thought of as inserted in some correlator, possibly with other fields inserted.

The first example of OPE is the OPE of $T$ with a primary operator $\Phi$, that we can infer from equation (3.28):

$$
T(z) \Phi(w, \bar{w}) \sim \frac{h}{(z-w)^{2}} \Phi(w, \bar{w})+\frac{1}{z-w} \partial_{w} \Phi(w, \bar{w}) .
$$

What can we say about the OPE of $T$ with a quasi-primary operator $\Phi$ ? Under an infinitesimal global conformal transformation $z \mapsto z+\alpha z$, we have

$$
\delta \Phi(z, \bar{z})=-(\alpha z \partial \Phi(z, \bar{z})+\bar{\alpha} \bar{z} \bar{\partial} \Phi(z, \bar{z})+(h \alpha+\bar{h} \bar{\alpha}) \Phi(z, \bar{z})) .
$$

On the other hand the Ward identity (3.33) with $\varepsilon(z)=\alpha z$ yields

$$
\begin{aligned}
\delta\langle\Phi(z, \bar{z})\rangle & =-\frac{1}{2 \pi i} \oint \mathrm{d} w \alpha w\langle T(w) \Phi(z, \bar{z})\rangle+\frac{1}{2 \pi i} \oint \mathrm{d} \bar{w} \bar{\alpha} \bar{w}\langle\bar{T}(\bar{w}) \Phi(z, \bar{z})\rangle \\
& =-\alpha\left\langle\{T \Phi\}_{2}(z, \bar{z})+z\{T \Phi\}_{1}(z, \bar{z})\right\rangle+\bar{\alpha}\left\langle\{\bar{T} \Phi\}_{2}(z, \bar{z})+\bar{z}\{\bar{T} \Phi\}_{1}(z, \bar{z})\right\rangle .
\end{aligned}
$$

The operators $\{T \Phi\}_{n}$ are thus given by

$$
\begin{array}{ll}
\{T \Phi\}_{1}=\partial \Phi, & \{T \Phi\}_{2}=h \Phi \\
\{\bar{T} \Phi\}_{1}=\bar{\partial} \Phi, & \{\bar{T} \Phi\}_{2}=\bar{h} \Phi .
\end{array}
$$

In conclusion, the most general form of the OPE of $T$ with a quasi-primary field $\Phi$ is of the form

$$
T(z) \Phi(w, \bar{w}) \sim \cdots+\frac{h}{(z-w)^{2}} \Phi(w, \bar{w})+\frac{\partial \Phi(w, \bar{w})}{z-w},
$$

and similarly for $\bar{T}$, where the dots denote extra terms that diverge like $(z-w)^{-n}$ with $n \geq 3$. When all these extra terms vanish, (3.48) implies that $\Phi$ is primary.

Since $T$ is itself a quasi-primary (of weights $(h, \bar{h})=(2,0)$ ), we can look at the case where $\Phi$ is taken to be $T$ in formula (3.54). It can be shown that in this case, thanks to unitarity and using a dimensional argument, the only possible terms in addition to those already shown in (3.54) is proportional to $(z-w)^{-4}$. As a consequence, the TT OPE is

$$
T(z) T(w) \sim \frac{c / 2}{(z-w)^{4}}+\frac{2 T(w)}{(z-w)^{2}}+\frac{\partial T(w)}{z-w},
$$


for some number $c$, called the (holomorphic) central charge of the CFT. The origin of this name will be explained in the next subsection.

We similarly have, considering the anti-holomorphic operator $\bar{T}$, that

$$
\bar{T}(\bar{z}) \bar{T}(\bar{w}) \sim \frac{\bar{c} / 2}{(\bar{z}-\bar{w})^{4}}+\frac{2 \bar{T}(\bar{w})}{(\bar{z}-\bar{w})^{2}}+\frac{\bar{\partial} T(\bar{w})}{\bar{z}-\bar{w}},
$$

where again $\bar{c}$ is a number, called the anti-holomorphic central charge.

Using (3.43) with $\Phi$ replaced by $T$ and (3.55), we find that under any holomorphic conformal transformation $z \mapsto z+\varepsilon(z)$ we have

$$
\delta_{\varepsilon} T=-\frac{c}{12} \partial^{3} \varepsilon-2 T \partial \varepsilon-\varepsilon \partial T .
$$

Comparing with (3.21), we see that except when $c=0, T$ is not a primary field. It is a nice exercise to show that the finite transformation $T \mapsto T^{\prime}$ coming from the infinitesimal transformation (3.57) is, for $z \mapsto w(z)$, given by

$$
T^{\prime}(w)=\left(\frac{\mathrm{d} w}{\mathrm{~d} z}\right)^{-2}\left(T(z)-\frac{c}{12}\{w ; z\}\right),
$$

where $\{w ; z\}$ is called the Schwarzian derivative of $w$ with respect to $z$ and is defined by

$$
\{w ; z\} \equiv \frac{\mathrm{d}^{3} w / \mathrm{d} z^{3}}{\mathrm{~d} w / \mathrm{d} z}-\frac{3}{2}\left(\frac{\mathrm{d}^{2} w / \mathrm{d} z^{2}}{\mathrm{~d} w / \mathrm{d} z}\right)^{2} .
$$

\subsubsection{Virasoro algebra}

Now that we have introduced the concept of OPE, it is straightforward to find the algebra satisfied by the operators $L_{n}, \bar{L}_{m}$ using formula (3.39). The result reads

$$
\begin{aligned}
& {\left[L_{n}, L_{m}\right]=(n-m) L_{m+n}+\frac{c}{12} n\left(n^{2}-1\right) \delta_{m+n, 0},} \\
& {\left[L_{n}, \bar{L}_{m}\right]=0,} \\
& {\left[\bar{L}_{n}, \bar{L}_{m}\right]=(n-m) \bar{L}_{m+n}+\frac{\bar{c}}{12} n\left(n^{2}-1\right) \delta_{m+n, 0} .}
\end{aligned}
$$

Let us compare algebra (3.60) with the classical algebra given in (3.13). As in the classical case, the two different sets of generators $L_{m}$ and $\bar{L}_{m}$ corresponding to holomorphic and anti-holomorphic transformations (respectively) close to commuting subalgebras, known as the Virasoro algebra and defined by (3.60a) for the holomorphic part. The Virasoro algebra differs from the Witt algebra by an extra term, proportional to $c$ and commuting with all other operators; this term is called a central term, and this is the reason why $c$ is called the central charge. The appearance of a central extension, when going from the classical to the quantum theory, should not be surprising. Central extensions generically appear when the algebra for the operators in the quantum theory is considered, as a consequence of the fact that it is only representations up to a phase that matter in the quantum theory. It would be however much more surprising to find central extensions in purely classical field theories, and this can actually happen (see e.g. [10]). 


\subsection{Trace anomaly}

When a two-dimensional CFT is deformed to a curved space-time, one should expect that conformal invariance is broken since the space-time curvature introduces explicitly a length scale, the curvature radius. In this section we make this statement more precise by computing the trace anomaly, ${ }^{6}$ that is the failure for the trace of the energy-momentum tensor to have a vanishing vacuum expectation value, $\left\langle T^{\mu}{ }_{\mu}\right\rangle \neq 0$.

Let us start by some general considerations. The quantity $\left\langle T^{\mu}{ }_{\mu}\right\rangle$ has dimension on length ${ }^{-2}$, it must be local and scalar under rotations. As we have no dimensionful parameter in the theory, the only possibility is that $\left\langle T^{\mu}{ }_{\mu}\right\rangle$ is proportional to the Ricci scalar $R(g)$ of the metric $g$. Thus we must have ${ }^{7}$

$$
\left\langle T^{\mu}{ }_{\mu}\right\rangle=a R,
$$

for some dimensionless constant $a$. Our goal is now to find the precise value of $a$. To this end, we will compare to first order around flat space the transformation properties of both sides of (the derivative of) (3.61) under an infinitesimal conformal transformation composed with a suitably chosen diffeomorphism.

We use the usual complex coordinates $(z, \bar{z})$ and start with the operator relation

$$
T_{z \bar{z}}=\frac{a}{2} g_{z \bar{z}} R
$$

Applying the operator $\nabla_{z}$ to equation (3.62) yields

$$
\nabla^{\bar{z}} T_{z \bar{z}}=\frac{a}{2} \partial_{z} R
$$

where we used the fact that $\nabla_{z}=g_{z \bar{z}} \nabla^{\bar{z}}$. Using the conservation law (valid upon taking the expectation value) $\nabla_{\mu} T^{\mu v}=0$, we deduce that

$$
\nabla^{z} T_{z z}=-\frac{a}{2} \partial_{z} R
$$

We now consider the variation of both sides of equation (3.64) under the composition of the following two transformations:

1. A conformal transformation $z \mapsto z+\varepsilon(z)$. The jacobian of this map is

$$
\left|\frac{\partial \mathbf{x}^{\prime}}{\partial \mathbf{x}}\right|=1+\partial \varepsilon
$$

and the component $T_{z z}$ of the energy-momentum tensor varies according to

$$
\delta_{\varepsilon} T_{z z}=-\frac{1}{2 \pi} \delta_{\varepsilon} T=\frac{c}{24 \pi} \partial^{3} \varepsilon+\frac{1}{\pi} T \partial \varepsilon+\frac{\varepsilon}{2 \pi} \partial T
$$

where we used equation (3.57).

\footnotetext{
${ }^{6}$ The trace anomaly is also know as the conformal anomaly.

${ }^{7} \mathrm{~A}$ word of caution: the arguments given here does not constitute a complete proof of equation (3.61). For a detailed proof, we refer the reader to the literature, see e.g. [3].
} 
2. A diffeomorphism $z \mapsto z-\varepsilon(z)$, under which the component $T_{z z}$ of the energy-momentum tensor varies according to

$$
\delta_{-\varepsilon} T_{z z}=-\frac{1}{\pi} T \partial \varepsilon-\frac{\varepsilon}{2 \pi} \partial T,
$$

where we simply used the fact that the energy-momentum tensor is a tensor under arbitrary diffeomorphisms.

Under the composition of the two above transformations, the net effect on $T_{z z}$ is simply

$$
\delta_{\mathrm{tot}} T_{z z}=\frac{c}{24 \pi} \partial^{3} \varepsilon
$$

Going to the conformal gauge $g_{\mu \nu}=e^{2 \omega} \delta_{\mu \nu}$ and working to first order in $\omega$, we find that the variation of the left hand side of (3.64) is given by

$$
\delta_{\mathrm{tot}} \nabla^{z} T_{z z}=\frac{c}{12 \pi} \bar{\partial} \partial^{3} \varepsilon
$$

Let us now determine the effect on $R$ of the same transformation. The net effect on the coordinates is the identity, while the net effect on the metric is an infinitesimal Weyl rescaling,

$$
g \mapsto(1+\partial \varepsilon) g .
$$

The effect of an arbitrary Weyl rescaling $g \mapsto \tilde{g}=e^{2 \sigma} g$ on the Ricci scalar is straightforward to work out (see e.g. in [11] for a proof of this formula generalised to any dimension) and reads

$$
\sqrt{\tilde{g}} R(\tilde{g})=\sqrt{g}\left(R(g)-2 \nabla_{g}^{2} \sigma\right),
$$

where $\nabla_{g}$ is the covariant derivative compatible with the metric $g$. In the case of the infinitesimal Weyl rescaling (3.70), we find using formula (3.71) the following expression for the variation $\delta R$ of the Ricci scalar:

$$
\delta R=-R \partial \varepsilon-\nabla^{2} \partial \varepsilon
$$

Moreover, in the conformal gauge $g_{\mu \nu}=e^{\omega} \delta_{\mu v}$ and in complex coordinates $(z, \bar{z})$, the curved laplacian $\nabla^{2}$ reads

$$
\nabla^{2}=4 e^{-2 \omega} \partial_{z} \partial_{\bar{z}}
$$

Plugging (3.73) into (3.72) and working at first order in $\omega$ we find, for the net variation of the right hand side of (3.64),

$$
-\frac{a}{2} \delta_{\mathrm{tot}} \partial_{z} R=2 a \partial^{3} \bar{\partial} \varepsilon
$$

Finally, comparing equation (3.69) and (3.74), we find $a=c /(24 \pi)$. Our final result is thus

$$
\left\langle T_{\mu}^{\mu}\right\rangle=\frac{c}{24 \pi} R
$$

\section{Acknowledgements}

Many thanks to Blagoje Oblak for his careful reading of the first version of these notes. The author is a Research Fellow of the Belgian Fonds de la Recherche Scientifique-FNRS. 


\section{References}

[1] M. Moskovic and D. Redigolo, a- and c-theorems, Unpublished.

[2] A. Sfondrini, An introduction to universality and renormalization group techniques, arXiv: 1210.2262 [hep-th].

[3] P. Di Francesco, P. Mathieu and D. Sénéchal, Conformal field theory, Springer, New York 1997.

[4] P. H. Ginsparg, Applied Conformal Field Theory, hep-th/9108028.

[5] S. Coleman, Aspects of Symmetry, USA: Cambridge U. Press 1985.

[6] J. Polchinski, String theory. Vol. 1: An introduction to the bosonic string, Univ. Pr., Cambridge UK, 1998.

[7] D. Tong, String Theory, arXiv:0908.0333 [hep-th].

[8] J. Polchinski, String theory. Vol. 2: Superstring theory and beyond, Univ. Pr., Cambridge UK 1998.

[9] J. Polchinski, Scale And Conformal Invariance In Quantum Field Theory, Nucl. Phys. B 303 (1988) 226.

[10] J. D. Brown and M. Henneaux, Central Charges in the Canonical Realization of Asymptotic Symmetries: An Example from Three-Dimensional Gravity, Commun. Math. Phys. 104 (1986) 207.

[11] M. Nakahara, Geometry, topology and physics, Taylor \& Francis, Boca Raton 2003. 